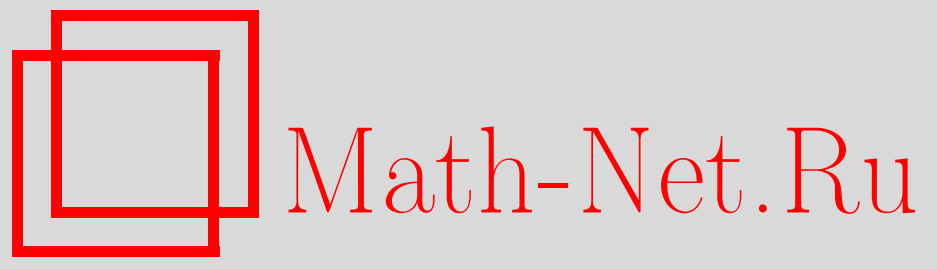

Г. И. Ивченко, С. А. Хонов, Статистическое оценивание состава конечной совокупности, Дискрет. матем., 1996, том 8, выпуск 1, 3-40

DOI: https://doi.org/10.4213/dm511

Использование Общероссийского математического портала Math-Net.Ru подразумевает, что вы прочитали и согласны с пользовательским соглашением http://www . mathnet.ru/rus/agreement

Параметры загрузки:

IP : 3.93.64.190

26 апреля 2023 г., 15:52:17

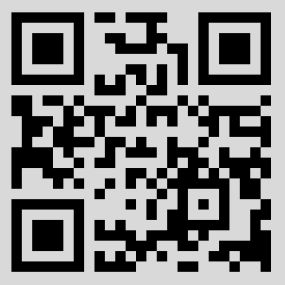




\title{
Статистическое оценивание состава конечной совокупности
}

\author{
(c) 1996 г. Г. И. Ивченко, С. А. Хонов
}

\begin{abstract}
Дается обзор последних результатов по проблеме оценивания состава конечной совокупности, примыкающих к задачам размещения частиц по ячейкам; при этом рассматриваются случаи как однородной, так и расслоенной. совокупностей. В рамках обобщенной схемы выбора дается сравнительный анализ результатов, получаемых с использованием принципов достаточности и правдоподобия. Излагаются также результаты, получаемые с помощью асимптотического подхода. Акцент делается на получении оптимальных (асимптотически оптимальных) выводов как при точечном, так и при доверительном оценивании. Обсуждаются возможные продолжения исследований.
\end{abstract}

\section{1. Введение}

Оценивание неизвестного размера конечной совокупности $U$, а также размеров составляющих ее слоев (классов) в случаях, когда $U$ - расслоенная совокупность (кратко - оценивание состава $U$ ), представляет собой традиционную и интенсивно разрабатываемую область прикладного статистического анализа, имеющую широкую сферу разнообразных практических приложений: выборочное обследование биологических популяций, текстов в лингвистике, статистический контроль качества промышленной продукции, социологические и медицинские исследования и т.д.

Имеется огромное число работ как теоретических, так и сугубо прикладного характера, посвященных этой проблематике, которые различаются конкретизациями структуры исследуемой совокупности $U$, соответствующего выборочного плана (выборочного механизма для получения исходной статистической информации об $U$ ) и цели исследования (а следовательно, характером и масштабностью результатов). Одно перечисление подобных работ заняло бы слишком много места (их список только за последние несколько лет насчитывает несколько десятков наименований), и мы не ставим здесь цель объять необъятное, отсылая интересующихся историей вопроса к монографиям Ю. К. Беляева [1], В. Г. Воинова и М. С. Никулина [3], а также к обзорам В. А. Иванова, Г. И. Ивченко и Ю. И. Медведева [4] и Себера [37], в которых в той или иной мере подводятся итоги соответствующих этапов (в определенных направлениях) развития этой тематики. Наша цель более конкретна (и в значительной степени коррелирована с научными интересами и результатами авторов статьи) - 
дать аналитический обзор соответствующих математических результатов, примыкающих к задачам размещения частиц по ячейкам и полученных, в основном, в последнее время (при этом в обзор включен и ряд еще не опубликованных результатов), а также наметить некоторые возможные продолжения исследований в данной области и, тем самым, как мы надеемся, стимулировать дальнейший интерес к этой проблематике.

\section{2. Модель}

В задачах выборочного обследования конечных совокупностей, связанных с оцениванием ее состава, ключевыми являются следующие три элемента: структура исследуемой совокупности, тип выборочного механизма, используемого для получения исходных статистических данных, и объект оценивания. Эти элементы в основном определяют применяемые методы исследования и получаемые в итоге результаты, поэтому остановимся на них подробнее.

\section{1. Структура совокупности}

В общем случае элементы исследуемой конечной совокупности $U$ разбиты по некоторому признаку (пол, возраст, сорт, доход, емкость и т.п.) на некоторое известное число $k$ классов (иногда говорят - слоев) $U_{1}, \ldots, U_{k}$ так, что

$$
\bigcup_{j=1}^{k} U_{j}=U, \quad U_{i} \cap U_{j}=\varnothing, \quad i \neq j
$$

в таких случаях говорят о расслоенной ( $k$-слойной) совокупности; случай $k=1$ соответствует нерасслоенной, или однородной совокупности. Пусть $N=|U|$ обозначает число всех элементов $U$ (ее размер), $N_{j}=\left|U_{j}\right|$ - размер $j$-го слоя, $j=1, \ldots, k$, тогда параметрический вектор $\bar{N}=\left(N_{1}, \ldots, N_{k}\right)$ называется составом совокупности $U$ (для однородной совокупности состав и размер означают одно и то же).

В задачах, о которых идет речь, состав $\bar{N}$ исследуемой совокупности является неизвестным параметром, о котором может иметься лишь некоторая частичная априорная информация, заданная множеством $\mathcal{N}=\{\bar{N}\}$ априори возможных его значений (всегда далее предполагается, что совокупность $U$ непустая, т.е. $\left.N=N_{1}+\ldots+N_{k}>0\right)$. Структура этого параметрического множества $\mathcal{N}$ в разных задачах может быть различной, но в естественных ситуациях она, как правило, определяется линейными ограничениями типа $N^{\prime} \leqslant N \leqslant N^{\prime \prime}, N_{j}^{\prime} \leqslant N_{j} \leqslant N_{j}^{\prime \prime}$, $j=1, \ldots, k$, где границы $N^{\prime}, N^{\prime \prime}, N_{j}^{\prime}, N_{j}^{\prime \prime}, j=1, \ldots, k$, или часть из них априори заданы (здесь $N^{\prime}>0, N^{\prime \prime} \leqslant \infty, N_{j}^{\prime} \geqslant 0, N_{j}^{\prime \prime} \leqslant \infty$, причем крайние случаи фактически означают отсутствие соответствующих ограничений). В качестве более конкретных примеров можно выделить следующие типичные случаи:

$\mathcal{N}^{(1)}=\mathcal{N}_{N^{0}}=\left\{\bar{N}=\left(N_{1}, \ldots, N_{k}\right): N=N_{1}+\ldots+N_{k}=N^{0}\right\}$, где $N^{0}$ - заданный общий размер совокупности $U=\bigcup_{j=1}^{k} U_{j}$; этот случай характерен для задач выборочного контроля качества промышленной продукции; 
$\mathcal{N}^{(2)}=\bigcup_{i \geqslant 1} \mathcal{N}_{i}=\{\bar{N}: N>0\}$, это случай огутствия какой-либо априорной информации и он характерен для задач обследования биологических популяций;

$\mathcal{N}^{(3)}=\mathcal{N}_{m, M}=\bigcup_{i=m}^{M} \mathcal{N}_{i}=\{\bar{N}: m \leqslant N \leqslant M\}$, т.е. априори известно, что размер совокупности находится в пределах между заданными $m$ и $M$, это промежуточный случай между двумя предшествующими случаями);

$\mathcal{N}^{(4)}=\mathcal{N}_{i}^{+} \equiv\left\{\bar{N}: N=i, N_{j}>0, j=1, \ldots, k\right\}, i \geqslant k$, т.е. априори известно, что совокупность состоит из $i$ элементов и все со $c$ авляющие ее $k$ классов непусты;

$\mathcal{N}^{(5)}=\mathcal{N}^{+}=\bigcup_{i \geqslant k} \mathcal{N}_{i}^{+}$, т.е. априори лишь известно, что все классы $k$-слойной совокупности непусты.

Структура параметрического множества $\mathcal{N}$ играет существенную роль для тех методов оценивания, в которых используется свойство полноты соответствующей достаточной статистики, о чем подробнее будет идти речь ниже. Описанная конструкция позволяет охватить большое разнообразие возникающих в приложениях ситуаций.

\section{2. Выборочный план}

В приложениях для получения исходных данных (исходной статистической информации об исследуемой совокупности $U$ ) часто используются классические схемы повторной и бесповторной выборок. Мы будем работать в основном в рамках следующей более общей выборочной процедуры, называемой обобщенной схемой выбора (OCB), когда из совокупности $U$ последовательно извлекаются $s$ независимых бесповторных выборок некоторых объемов $m_{1}, \ldots, m_{s}$ соответственно, при этом в $i$-й выборке любая из $\left(\begin{array}{c}N \\ m_{i}\end{array}\right)$ различных комбинаций элементов $U$ по предположению может появиться с равной вероятностью (практически независимость выборок обеспечивается возвращением в совокупность всех извлеченных на предыдущем этапе элементов после их метки). Таким образом, число исходов такого статистического эксперимента есть

$$
\Psi_{s}(N)=\prod_{i=1}^{s}\left(\begin{array}{c}
N \\
m_{i}
\end{array}\right)
$$

и все они по условию равновероятны. Выборочный план (ВП), соответствующий $\mathrm{OCB}$ с параметрами $m_{1}, \ldots, m_{s}$ и общим ресурсом испытаний $n=m_{1}+\ldots+$ $m_{s}$, В дальнейшем обозначается символом $\Pi_{n}\left(m_{1}, \ldots, m_{s}\right)$. В случае, когда все частные выборки имеют одинаковый объем $m$, соответствующий ВП будем обозначать $\Pi_{n}(s, m)$ (так что здесь $n=s m$ ); тогда $\Pi_{n}(n, 1)=\Pi_{n}$ есть план простой повторной, а $\Pi_{n}(1, n)=\bar{\Pi}_{n}$ при $n \leqslant N$ простой бесповторной выборок объема $n$. В дальнейшем используется также обозначение $m=\max _{1 \leqslant i \leqslant s} m_{i}$ для объема максимальной частной выборки и априори предполагается, что $N \geqslant m$. Подчеркнем, что ОСВ включает как частные случаи схемы простых повторной (при 
$\left.m_{i}=1, i=1, \ldots, s\right)$ и бесповторной (при $s=1$ ) выборок и относится к классу выборочных планов с фиксированным общим числом наблюдений.

Получаемые при ОСВ данные мы будем записывать в виде $\bar{\mu}=\left(\mu_{j r}, j=\right.$ $1, \ldots, k ; r=1, \ldots, s)$, где $\mu_{j r}$ означает число элементов из класса $U_{j}$, наблюденных ровно $r$ раз каждый (таким образом, в данном случае не учитывается порядок извлечения элементов, а также какая-нибудь другая дополнительная информация, помимо меток, характеризующих принадлежность элементов соответствующим классам).

Другой популярный в литературе класс выборочных планов составляют различные схемы выбора последовательного типа со случайным числом наблюдений, определяемым тем или иным правилом остановки (ключевой элемент плана). Такие планы для некоторых правил остановки также обсуждаются ниже (см. п.6.6).

\section{3. Объект оценивания}

Чаще всего целью исследования является оценивание (точное и доверительное) общего размера $N$ совокупности $U$ (когда он неизвестен), а также всего вектора состава $\bar{N}=\left(N_{1}, \ldots, N_{k}\right)$ (в случае $k$-слойной совокупности) или отдельных его компонент. В общем случае интерес представляет произвольная параметрическая функция $\tau(\bar{N})$, в такой постановке прежде всего возникает задача описания класса оцениваемых функций. Могут также представлять интерес конкретные параметрические функции, отличные от тождественной (например, $\tau(\bar{N})=N^{-1}$ ), или конкретные классы параметрических функций. Конечно, желательно иметь общий результат, но он в ряде случаев трудно достижим; с примерами тех и других ситуаций мы еще встретимся.

Отметим, наконец, некоторые общие моменты, связанные с методами исследования обсуждаемой модели (подробнее об этом речь будет идти ниже). При ОСВ достаточной статистикой для параметра $\bar{N}$ является вектор

$$
\bar{\eta}=\left(\eta_{1}, \ldots, \eta_{k}\right)
$$

где

$$
\eta_{j}=\sum_{r=1}^{s} \mu_{j r}
$$

есть число различных элементов класса (слоя) $U_{j}, j=1, \ldots, k$, зарегистрированных во всех наблюдениях, причем в зависимости от структуры параметрического множества $\mathcal{N}$ в ряде случаев достаточная статистика обладает свойством полноты. Это позволяет в соответствующих случаях построить оптимальные (т.е. с минимальной дисперсией) несмещенные оценки как для самого параметра $\bar{N}$, так и для определенных классов параметрических функций $\tau(\bar{N})$. В то же время достаточная статистика $\bar{\eta}$ не всегда полна, и тем самым, оптимальные несмещенные оценки не всегда существуют. С другой стороны, даже в тех случаях, когда такие оценки существуют, принцип достаточности приводит к сложным для практических вычислений формулам. В свете этих обстоятельств в рассматриваемой проблематике более предпочтительным, на наш взгляд, является принцип правдоподобия, приводящий к оценкам максимального правдоподобия (ОМП) $\hat{N}=\left(\hat{N}_{1}, \ldots, \hat{N}_{k}\right)$ для параметра $\bar{N}$ и $\hat{\tau}=\tau(\hat{N})$ для параметрических 
функций $\tau(\bar{N})$, где по определению значение ОМП $\hat{N}$ при наблюдении $\bar{\eta}=\bar{l}$ определяется формулой

$$
\hat{N}(\bar{l})=\arg \max _{\bar{N} \in \mathcal{N}} \mathbf{P}_{\bar{N}}(\bar{\eta}=\bar{l}) .
$$

Обсуждению особенностей этих двух методов оценивания состава $\bar{N}$ совокупности $U$ при ОСВ и посвящена в основном настоящая работа.

В заключение укажем на связь рассматриваемых задач с задачами о случайных размещениях [19]. Именно, можно представлять себе, что имеется $N$ ячеек, разбитых на $k$ непересекающихся групп по $N_{1}, \ldots, N_{k}$ ячеек соответственно, в которых независимо и равновероятно размещаются $s$ комплектов частиц объемов $m_{1}, \ldots, m_{s}$ (все $\left(\begin{array}{c}N \\ m_{i}\end{array}\right)$ вариантов размещения частиц $i$-го комплекта равновероятны). Предполагается, что вектор $\bar{N}=\left(N_{1}, \ldots, N_{k}\right)$ неизвестен, а наблюдению доступны лишь заполнения непустых ячеек, и по этой информации требуется сделать те или иные статистические выводы о неизвестном значении $\bar{N}$. Отмеченное соответствие позволяет использовать в рассматриваемой проблематтике хорошо развитую для схем размещения современную вероятностную теорию, особенно в ее асимптотической (при $N \rightarrow \infty$ ) части.

\section{3. Принцип достаточности}

Рассмотрим, что дает в обсуждаемой проблематике принцип достаточности.

\section{1. Достаточная статистика и ее полнота}

Ответ на ключевой вопрос, что является достаточной статистикой в рассматриваемой модели и каково ее распределение, содержится в следующем утверждении [22].

Теорема 1. При $В \Pi \Pi_{n}\left(m_{1}, \ldots, m_{s}\right)$ достаточной статистикой для вектора состава $\bar{N}$ совокупности $U=\bigcup_{j=1}^{k} U_{j}$ является вектор $\bar{\eta}=\left(\eta_{1}, \ldots, \eta_{k}\right)$, где $\eta_{j}$ есть число различных наблюдавшихся әлементов слоя $U_{j}, j=1, \ldots, k$; распределение $u$ моменты достаточной статистики имеют вид

$$
\begin{aligned}
\mathbf{P}_{\bar{N}}(\bar{\eta}=\bar{l}) & =\Psi_{s}^{-1}(N) \prod_{j=1}^{k}\left(\begin{array}{c}
N_{j} \\
l_{j}
\end{array}\right) \Delta^{l_{1}+\ldots+l_{k}} \Psi_{s}(0), \quad \bar{l}=\left(l_{1}, \ldots, l_{k}\right) \in \mathcal{L}_{m, n}(\bar{N}), \\
\mathbf{E}_{\bar{N}} \prod_{j=1}^{k}\left(\eta_{j}\right)_{i_{j}} & =\Psi_{s}^{-1}(N) \prod_{j=1}^{k}\left(N_{j}\right)_{i_{j}} \Delta^{i_{1}+\ldots+i_{k}} \Psi_{s}\left(N-i_{1}-\ldots-i_{k}\right),
\end{aligned}
$$

где $\Delta$ - оператор конечной разности: $\Delta f(x)=f(x+1)-f(x)$;

$$
\begin{aligned}
& (a)_{j}=a(a-1) \ldots(a-j+1), \quad j>0, \quad(a)_{0}=1, \\
\mathcal{L}_{m, n}(\bar{N})= & \left\{\bar{l}: 0 \leqslant l_{j} \leqslant N_{j}, j=1, \ldots, k, m \leqslant l_{1}+\ldots+l_{k} \leqslant \min (n, N)\right\} .
\end{aligned}
$$


Доказательство этой теоремы проводится с помощью обычных для схем размещения комбинаторных рассуждений. Именно, в данном случае правдоподобие данных, т.е. вероятность $\mathbf{P}_{\bar{N}}(\bar{\mu}=\bar{l}), \bar{l}=\left(l_{j r}, j=1, \ldots, k, r=1, \ldots, s\right)$, представляется в виде

$$
\Psi_{s}^{-1}(N) \prod_{j=1}^{k}\left(\begin{array}{c}
N_{j} \\
l_{j}
\end{array}\right) A(\bar{l})
$$

где

$$
l_{j}=\sum_{r=1}^{s} l_{j r}
$$

есть совместное с событием $\{\bar{\mu}=\bar{l}\}$ значение статистики

$$
\eta_{j}=\sum_{r=1}^{s} \mu_{j r}, \quad j=1, \ldots, k
$$

а комбинаторный множитель $A(\bar{l})$, определяющий число различных способов формирования выборки соответствующего состава из отобранных $l_{i}$ элементов слоя $U_{i}, i=1, \ldots, k$, от параметра $\bar{N}$ уже не зависит. Отсюда по критерию факторизации следует достаточность статистики $\bar{\eta}$.

Вывод формулы (2) также стандартен для задач размещения частиц по ячейкам; технические детали для некоторых частных случаев можно найти в [11] (случай простой повторной выборки) и $[15,19]$ (случай однородной совокупности). Формула (3) для моментов непосредственно следует из (2) с использованием известных свойств оператора $\Delta$, в частности, формулы

$$
\Delta^{n} f(x)=\sum_{j=0}^{n}(-1)^{n-j}\left(\begin{array}{l}
n \\
j
\end{array}\right) f(x+j)
$$

Следующий вопрос, имеющий принципиальное значение для задач оценивания, - это вопрос о полноте достаточной статистики. В рассматриваемом случае ответ на него неоднозначен и зависит от структуры параметрического множества $\mathcal{N}$ модели. Как известно, критерием полноты статистики $\bar{\eta}$ является единственность несмещенной оценки нуля в классе статистик вида $\varphi(\bar{\eta})$, т.е. условие

$$
\mathbf{E}_{\bar{N}} \varphi(\bar{\eta})=0 \quad \forall \bar{N} \in \mathcal{N} \quad \Longrightarrow \quad \varphi(\bar{l})=0 \quad \forall \bar{l} \in \bigcup_{\bar{N} \in \mathcal{N}} \mathcal{L}_{m, n}(\bar{N})
$$

Переписав левую часть (4) с учетом (2) в виде

$$
\sum_{\bar{l} \in \mathcal{L}_{m, n}(\bar{N})} a_{\bar{N}}(\bar{l}) \varphi(\bar{l})=0 \quad \forall \bar{N} \in \mathcal{N}
$$

где

$$
a_{\bar{N}}(\bar{l})=\prod_{j=1}^{k}\left(\begin{array}{c}
N_{j} \\
l_{j}
\end{array}\right) \Delta^{l_{1}+\ldots+l_{k}} \Psi_{s}(0),
$$

мы видим, что это система линейных однородных уравнений относительно $\{\varphi(\bar{l})\}$, поэтому условие полноты в данном случае эквивалентно условию единственности (нулевого) решения системы (5). Необходимым условием для этого 
является, очевидно, неравенство $\left|L_{m, n}\right| \leqslant|\mathcal{N}|$ (число неизвестных не должно превышать числа уравнений). Для некоторых типов множеств $\mathcal{N}$ это условие является одновременно и достаточным. В частности, это будет так для множеств линейчатой структуры, т.е. когда

$$
\mathcal{N}=\mathcal{N}_{m, M}=\bigcup_{i=m}^{M}\{\bar{N}: N=i\}
$$

при некотором $M \geqslant m$; в случае простой повторной выборки $\Pi_{n}$ этот факт установлен в [5], для произвольного ВП $\Pi_{n}\left(m_{1}, \ldots, m_{s}\right)$ и нерасслоенной совокупности $(k=1)$ в [15], а в общем случае в [22]. Если $\mathcal{N}=\left\{\bar{N}: N=N^{0}, N^{0}>m\right\}$, то статистика $\bar{\eta}$ полна лишь в случае одной бесповторной выборки, т.е. при ВП $\bar{\Pi}_{n}$ (в случае $k=2$ это хорошо известный результат для гипергеометрического распределения). Если же $s>1$, то статистика $\bar{\eta}$ свойством полноты уже не обладает: в этом случае существует свободная статистика, а именно, общее число различных наблюдавшихся элементов совокупности $\eta=\eta_{1}+\ldots+\eta_{k}$, ее распределение и моменты имеют вид [23]

$$
\begin{gathered}
\mathbf{P}_{\bar{N}}(\eta=l)=\Psi_{s}^{-1}\left(N^{0}\right)\left(\begin{array}{c}
N^{0} \\
l
\end{array}\right) \Delta^{l} \Psi_{s}(0), \quad m \leqslant l \leqslant n \wedge N^{0}, \\
\mathbf{E}_{\bar{N}}(\eta)_{i}=\Psi_{s}^{-1}\left(N^{0}\right)\left(N^{0}\right)_{i} \Delta^{i} \Psi_{s}\left(N^{0}-i\right), \quad i \geqslant 1 .
\end{gathered}
$$

Эти два случая задания параметрического множества $\mathcal{N}$, наиболее интересные для приложений, в основном и рассматривались в литературе; ниже они и будут объектом нашего внимания.

\section{2. Несмещенное оценивание}

Пусть $\tau(\bar{N})$ - подлежащая оцениванию параметрическая функция. Здесь мы ограничиваемся рассмотрением лишь несмещенных оценок функции $\tau(\bar{N})$ в тех или иных классах статистик и наша цель - построение наилучшей по критерию минимума дисперсии оценки, т.е. несмещенной оценки с (равномерно) минимальной дисперсией (НОМД) (термин равномерно, как это часто делается в литературе, мы для краткости опускаем). При наличии полной достаточной статистики (в нашем случае это статистика $\bar{\eta}$ ) задача построения такой оценки сводится, как известно, к выяснению условий разрешимости и отысканию при этих условиях явного вида решения уравнения несмещенности

$$
\mathbf{E}_{\bar{N}} \varphi(\bar{\eta})=\tau(\bar{N}) \quad \forall \bar{N} \in \mathcal{N} ;
$$

функция $\tau(\bar{N})$, для которой уравнение (7) имеет решение, называется при этом допускающей оценку. На этом пути получены следующие общие результаты.

Теорема 2. Если параметрическое мнохсество модели имеет вид $\mathcal{N}=\mathcal{N}_{m, M}$ при некотором $M, m \leqslant M \leqslant n$, то при $B \Pi \Pi_{n}\left(m_{1}, \ldots, m_{s}\right)$ НОМД существует для любой функции $\tau(\bar{N})$ и дается формулой

$$
\tau^{*}=\frac{1}{\Delta^{\eta} \Psi_{s}(0)} \sum_{i_{1}+\ldots+i_{k} \geqslant m} f\left(i_{1}, \ldots, i_{k}\right) \prod_{j=1}^{k}(-1)^{\eta_{j}-i_{j}}\left(\begin{array}{c}
\eta_{j} \\
i_{j}
\end{array}\right)
$$


или әквивалентной формулой

$$
\tau^{*}=\frac{1}{\delta^{\eta} \Psi_{s}(0)}\left(f(\overline{0}) \prod_{j=1}^{k} \Delta_{j}^{\eta_{j}} i_{j}-\sum_{i_{1}+\ldots+i_{k}<m} f\left(i_{1}, \ldots, i_{k}\right) \prod_{j=1}^{k}(-1)^{\eta_{j}-i_{j}}\left(\begin{array}{c}
\eta_{j} \\
i_{j}
\end{array}\right)\right)
$$

əде $f(\bar{N})=\tau(\bar{N}) \Psi_{s}(N)$ u $\Delta_{j}$ - оператор разности по $j$-ой переменной, $j=1, \ldots, k$.

Если жсе вне $\mathcal{N}$ функция $\tau(\bar{N})$ такова, что $f(\bar{N})=0$ при $0 \leqslant N<m$, в частности, если $\tau(\bar{N})$ конечна при всех $\bar{N}$, то формула (8) сводится к виду

$$
\tau^{*}=\Delta_{1}^{\eta_{1}} \ldots \Delta_{k}^{\eta_{k}} f(\overline{0}) / \Delta^{\eta} \Psi_{s}(0)
$$

Теорема 3. Пусть $\mathcal{N}=\mathcal{N}_{m, M}$ при некотором $M>n$. Тогда допускающими оченку являются лишь функиии вида $\tau(\bar{N})=f(\bar{N}) \Psi_{s}^{-1}(N)$, где $f(\bar{N})$ - многочлен степени $n$, удовлетворяющий при $n+1 \leqslant N \leqslant M$ условию

$$
\sum_{\substack{l_{1}+\ldots+l_{k} \leqslant n \\
i_{1}+\ldots+i_{k}<m}} f\left(i_{1}, \ldots, i_{k}\right) \prod_{j=1}^{k}(-1)^{l_{j}-i_{j}}\left(\begin{array}{c}
N_{j} \\
l_{j}
\end{array}\right)\left(\begin{array}{c}
l_{j} \\
i_{j}
\end{array}\right)=0
$$

и в этом случае НОМД $\tau^{*}$ дается формулой (8); если же $M>2 n$, то (10) сводится к условию $f(\bar{N})=0$ при $0 \leqslant N<m, u$ если $\tau(\bar{N})-$ такая функция, то НОМД для нее дается формулой (9).

Утверждения теорем 2 и 3 для нерасслоенных совокупностей $(k=1)$ получены в работе [15], для схемы простой повторной выборки $\Pi_{n}$ в [5], а для общего случая в [22].

Отметим некоторые интересные частные случаи этих результатов. Если $\tau(\bar{N})=\tau(N)$, т.е. функция зависит лишь от общего размера совокупности $N=$ $N_{1}+\ldots+N_{k}$, и является конечной, то ее НОМД имеет вид (при $N \leqslant n$ )

$$
\tau^{*}=\Delta^{\eta}\left(\tau(0) \Psi_{s}(0)\right) / \Delta^{\eta} \Psi_{s}(0)
$$

При $\tau(N)=N$ мы имеем отсюда соответствующий результат для оценивания $N=|U|$; впервые оценка $N$ для схемы простой повторной выборки $\Pi_{n}$

$$
N^{*}=\Delta^{\eta} 0^{n+1} / \Delta^{\eta} 0^{n}
$$

была получена Харрисом [30].

При отсутствии априорных ограничений на общий размер $N$ совокупности $U$ в схеме простой повторной выборки $\Pi_{n}$ НОМД существует для любой степени $N^{-j}$ при $j<n$, именно [5],

$$
\left(N^{-j}\right)^{*}=\Delta^{\eta} 0^{n-j} / \Delta^{\eta} 0^{n}
$$

для функций же от долей $\bar{\beta}=\left(\beta_{j}=N_{j} / N, \quad j=1, \ldots, k\right)$ вида

$$
\tau(\bar{\beta})=\prod_{j=1}^{k} \beta_{j}^{r_{j}}
$$


НОМД существует лишь при $r_{1}+\ldots+r_{k}=r \leqslant n$ и имеет вид

$$
\tau^{*}=\left.\left(\Delta^{\eta} 0^{n}\right)^{-1} \Delta_{1}^{\eta_{1}} \ldots \Delta_{k}^{\eta_{k}} x_{1}^{r_{1}} \ldots x_{k}^{r_{k}}\left(x_{1}+\ldots+x_{k}\right)^{n-r_{1}-\ldots-r_{k}}\right|_{\bar{x}=\overline{0}} .
$$

Пусть теперь множество $\mathcal{N}=\mathcal{N}_{N^{0}}$ при $N^{0}>m$. Тогда [23] при ВП $\bar{\Pi}_{n}$ (одна бесповторная выборка) статистика $\bar{\eta}$, как отмечалось выше, полна и НОМД для размеров классов имеют вид $N_{j}^{*}=\eta_{j} N^{0} / n, j=1, \ldots, k$.

В многовыборочной схеме $\Pi_{n}\left(m_{1}, \ldots, m_{s}\right)$ при $s>1$ статистика $\bar{\eta}$ не является полной, поэтому уравнение несмещенности (7) уже не обладает свойством единственности решения в классе всех функций от $\bar{\eta}$. Однако в ряде случаев такой единственности можно добиться за счет сужения класса оценок, в котором ищется решение. Именно, введем класс полиномиальных функций от статистики $\bar{\eta}$ :

$$
\mathcal{T}_{r}=\left\{T_{r}(\bar{\eta})=\sum_{i_{1}+\ldots+i_{k}=r} a_{i_{1} \ldots i_{k}}\left(\eta_{1}\right)_{i_{1}} \ldots\left(\eta_{k}\right)_{i_{k}}\right\}, \quad r \leqslant n \wedge N^{0},
$$

и будем искать в этом классе несмещенные оценки для функций вида

$$
\tau_{i_{1} \ldots i_{k}}(\bar{N})=\prod_{j=1}^{k}\left(N_{j}\right)_{i_{j}}
$$

Теорема 4 ([23]). При $B \Pi \Pi_{n}\left(m_{1}, \ldots, m_{s}\right)$ для любого $r \leqslant n \wedge N^{0}$ в классе $\mathcal{T}_{r}$ cyществует единственная несмещенная оченка функции $\tau_{i_{1} \ldots i_{k}}(\bar{N}) n p u i_{1}+\ldots+i_{k}=$ $i \leqslant r$, имеющая вид

$$
\tau_{i_{1} \ldots i_{k}}^{*}=\frac{(\eta-i)_{r-i} \Psi_{s}\left(N^{0}\right)}{\left(N^{0}-i\right)_{r-i} \Delta^{r} \Psi_{s}\left(N^{0}-r\right)} \prod_{j=1}^{k}\left(\eta_{j}\right)_{i_{j}}
$$

В частности, единственной несмещенной оценкой в классе $\mathcal{T}_{r}, r \leqslant n \wedge N^{0}$, для параметра $N_{j}$ является статистика

$$
T_{r j}^{*}(\bar{\eta})=\frac{(\eta-1)_{r-1} \Psi_{s}\left(N^{0}\right)}{\left(N^{0}-1\right)_{r-1} \Delta^{r} \Psi_{s}\left(N^{0}-r\right)} \eta_{j}
$$

дисперсия этой оценки растет с ростом $r$, поэтому простейшая (линейная) оченка

$$
T_{1 j}^{*}(\bar{\eta})=\frac{\Psi_{s}\left(N^{0}\right)}{\Delta \Psi_{s}\left(N^{0}-1\right)} \eta_{j}=\left(1-\prod_{i=1}^{s}\left(1-\frac{m_{i}}{N^{0}}\right)\right)^{-1} \eta_{j}
$$

является одновременно и наиболее точной среди оченок такого вида.

\section{3. Линейные оценки}

В ряде случаев могут оказаться полезными простые по структуре и поэтому удобные для быстрых расчетов несмещенные оценки, линейные по исходным данным $\bar{\mu}=\left\{\mu_{j r}\right\}$. Так, если совокупность $U$ нерасслоенная, и на ее размер 
не накладывается никаких априорнлх ограничений, то, как отмечалось выше, в схеме простой повторной выборки $\Pi_{n}$ НОМД существуют лишь для параметрических функций вида

$$
\tau(N)=\sum_{j=0}^{n-1} c_{j} N^{-j}
$$

и при этом

$$
\tau^{*}=\left(\Delta^{\eta} 0^{n}\right)^{-1} \sum_{j=0}^{n-1} c_{j} \Delta^{\eta} 0^{n-j} .
$$

В то же время для оценивания такой функции можно предложить простую несмещенную оценку, линейную пс $\mu_{1}, \ldots, \mu_{n}$ ( $\mu_{r}$ - число элементов $U$, повторившихся в выборке ровно $r$ раз), а именно [12],

$$
\tilde{\tau}=\sum_{j=0}^{n-1} c_{j} \sum_{r=1}^{n} \frac{(r)_{j+1}}{(n)_{j+1}} \mu_{r}
$$

В частности, для оценивания функции $\tau(N)=1 / N$ эти альтернативные оценки имеют вид (при $n \geqslant 2)$

$$
\left(\frac{1}{N}\right)^{*}=\frac{\Delta^{\eta} 0^{n-1}}{\Delta^{\eta} 0^{n}}, \quad \widetilde{\left(\frac{1}{N}\right)}=\frac{1}{n(n-1)} \sum_{r=2}^{n} r(r-1) \mu_{r} .
$$

При ВП $\Pi_{n}\left(m_{1}, \ldots, m_{s}\right)$ соответствующие оценки для $\tau(N)=1 / N$ принимают форму [15]

$$
\left(\frac{1}{N}\right)^{*}=\frac{\Delta^{\eta}\left(0^{-1} \Psi_{s}(0)\right)}{\Delta^{\eta} \Psi_{s}(0)}, \quad \widetilde{\left(\frac{1}{N}\right)}=\frac{\sum_{r=2}^{s} r(r-1) \mu_{r}}{\sum_{i \neq j} m_{i} m_{j}} .
$$

При этом указанные оценки $\tilde{\tau}$ являются единственными несмещенными оценками в классе линейных оценок.

Доказано также [12], что при ВП $\Pi_{n}(s, m)$ в классе линейных (по $\mu_{1}, \ldots, \mu_{s}$ ) оценок несмещенные оценки для $\tau(p), p=m / N$, существуют тогда и только тогда, когда $\tau(p)$ - полином степени $k \leqslant s-1 ;$ если

$$
\tau(p)=\sum_{j=0}^{k} c_{j} p^{j}
$$

то единственной такой оценкой является статистика

$$
\tilde{\tau}=\frac{1}{m} \sum_{r=1}^{s} \mu_{r} \sum_{j=0}^{k} c_{j} \frac{(r)_{j+1}}{(s)_{j+1}} .
$$

Приведем еще один результат такого типа для случая расслоенной совокупности [5]: если из совокупности

$$
U=\bigcup_{j=1}^{k} U_{j}
$$


извлечена простая повторная выборка объема $n$, то линейными по данным $\left\{\mu_{j r}\right\}$ несмещенными оценками долей классов $\beta_{j}=N_{j} / N, j=1, \ldots, k$, являются статистики

$$
\tilde{\beta}_{j}=\frac{1}{n} \sum_{r=1}^{n} r \mu_{j r}, \quad j=1, \ldots, k,
$$

в то время как НОМД имеют вид

$$
\beta_{j}^{*}=\left.\frac{1}{\Delta^{\eta} 0^{n}} \Delta_{1}^{\eta_{j}} \Delta_{2}^{\eta-\eta_{j}} x_{1}\left(x_{1}+x_{2}\right)^{n-1}\right|_{x_{1}=x_{2}=0}, \quad j=1, \ldots, k .
$$

\section{4. Принцип правдоподобия}

Как показано выше, НОМД в рассматриваемой модели не всегда существуют, а при их наличии они имеют весьма сложную структуру и поэтому трудоемки для практических расчетов, что неоднократно отмечалось в литературе (см., например, $[3,26])$. Поэтому разумной альтернативой к изложенной выше методике является метод максимального правдоподобия [33], определяемый в нашем случае соотношениями (1)-(2).

\section{1. Нерасслоенная совокупность}

Метод максимального правдоподобия в рассматриваемой проблематике ранее применялся в ряде работ, но в основном лишь в случае нерасслоенной совокупности $U$ (т.е. при $k=1$ ). Так, в монографии В. Феллера [21, гл.II, §6, пример (в)] обсуждается пример оценивания этим методом неизвестного числа $N$ рыб в озере по результатам двух независимых уловов $(s=2)$ и установлено, что искомая оценка имеет вид (в наших обозначениях) $\hat{N}=\left[m_{1} m_{2} /\left(m_{1}+m_{2}-\eta\right)\right]$, где $\eta$ есть число различных пойманных за оба улова рыб и $[a]$ обозначает целую часть $a$. В дальнейшем мы будем назынать ее оценкой Феллера. Подробное обсуждение свойств этой и связанных с ней оценок и предшествующая библио-

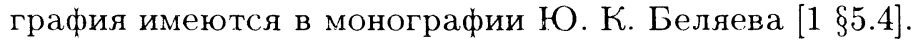

Схема простой повторной выборки $\Pi_{n}$ (все $N^{n}$ возможных вариантов выбора равновероятны) была проанализирована методом правдоподобия в [28], где установлено, что ОМП $\hat{N}=\hat{N}(\eta)(\eta$ - число различных элементов $U$ в выборке) при $1<\eta<n$ находится из условия

$$
S(\hat{N}, \eta)<\dot{n} \leqslant S(\hat{N}-1, \eta)
$$

где

$$
S(N, r)=\left(\ln \frac{N+1}{N+1-r}\right) / \ln \frac{N+1}{N}, \quad 1 \leqslant r \leqslant N
$$

если же $\eta=1$, то $\hat{N}=1$, а при $\eta=n$ ОМП не определена (формально $\hat{N}=\infty)$. В [28] приводится также таблица наименьших целых, больших или равных $S(r+j, r)$, для $r=1, \ldots, 50, j=1, \ldots, 20$, позволяющая находить $\hat{N}$ для тех случаев, когда значение статистики $\eta$ не превосходит 50. Асимптотические свойства этой оценки для больших выборок, включая и построение асимптотического доверительного интервала для $N$, основанного на $\hat{N}$, рассматривались в 
[32], а также в [14]. В частности, было установлено, что при условии $n, N \rightarrow \infty$, $n \asymp N$, ОМП $\hat{N}$ асимптотически находится по формуле $\hat{N}=n / a^{-1}(\eta / n)$, где $a^{-1}(t)$ есть обратная функция к $a(\alpha)=(1-\exp (-\alpha)) / \alpha, \alpha>0$. (Таблица значений функции $a^{-1}(t)$ приводится в п.6.4). Асимптотический (1- $\left.\delta\right)$-доверительный интервал для параметра $\alpha=n / N$ имеет вид

$$
\left(\hat{\alpha} \pm t_{\delta / 2} \sqrt{\hat{\alpha}^{3} /(n(\exp (\hat{\alpha})-1-\hat{\alpha}))}\right)
$$

где $\hat{\alpha}=n / \hat{N}$ и $t_{\delta / 2}$ обозначает верхнюю $\delta / 2$-гроцентиль нормального $\mathcal{N}(0,1)$ распределения; для самого же параметра $N$ соответствующий доверительный интервал имеет вид

$$
\left(\hat{N} \pm t_{\delta / 2} \sqrt{\hat{N} /(\exp (\hat{\alpha})-1-\hat{\alpha})}\right)
$$

Обобщение результатов (12) и (13) на случай $\mathrm{B \Pi} \Pi_{n}(s, m)$ проведено в [13], где установлено (с некоторыми нашими уточнениями), что ОМП $\hat{N}$ при $m<\eta<n$ находится из условия

$$
S_{m}(\hat{N}, \eta)<s \leqslant S_{m}(\hat{N}-1, \eta)
$$

где

$$
S_{m}(N, r)=\left(\ln \frac{N+1}{N+1-r}\right) / \ln \frac{N+1}{N+1-m}, \quad m \leqslant r \leqslant N ;
$$

если же $\eta=m$, по $\hat{N}=m$, а при $\eta=n$ оценка $\hat{n}$ не определена $(\hat{N}=\infty)$.

В качестве примеров решения неравенств (14) приведем значения оценки $\hat{N}=$ $\hat{N}(\eta)$ для некоторых значений параметров $s, m$ и статистики $\eta$ :

$$
\begin{array}{rlrlll}
s=2 & m=5: & \widehat{N}(6)=6 & \widehat{N}(7)=8 & \widehat{N}(8)=12 & \widehat{N}(9)=24 \\
& m=6: & \widehat{N}(7)=7 & \widehat{N}(8)=8 & \widehat{N}(9)=12 & \widehat{N}(10)=17 \\
& m=7: & \widehat{N}(8)=8 & \widehat{N}(9)=9 & \widehat{N}(10)=12 & \widehat{N}(11)=16 \\
m=8: & \widehat{N}(10)=10 & \widehat{N}(11)=12 & \widehat{N}(12)=15 & \widehat{N}(13)=21 \\
m=9: & \widehat{N}(11)=11 & \widehat{N}(12)=13 & \widehat{N}(13)=16 & \widehat{N}(14)=20 \\
m=10: & \widehat{N}(12)=12 & \widehat{N}(13)=14 & \widehat{N}(14)=16 & \widehat{N}(15)=19 \\
s=3 & m=5: & \widehat{N}(9)=10 & \widehat{N}(10)=13 & \widehat{N}(11)=16 & \widehat{N}(12)=23 \\
& m=6: & \widehat{N}(11)=13 & \widehat{N}(12)=15 & \widehat{N}(13)=19 & \widehat{N}(14)=24 \\
m=7: & \widehat{N}(12)=13 & \widehat{N}(13)=15 & \widehat{N}(14)=18 & \widehat{N}(15)=21
\end{array}
$$

В той же работе исследовано и асимптотическое поведение этой оценки в условиях $m, N \rightarrow \infty, 0<\lim m / N<1$ ( $s$ фиксировано) и, в частности, установлено, что асимптотически $\hat{N}=m / b_{s}^{-1}(\eta / m)$, где $b_{s}^{-1}(t)$ есть обратная функция к $b_{s}(p)=\left(1-q^{s}\right) / p, p=1-q \in(0,1)$. Асимптотическим $(1-\delta)$-доверительным интервалом для параметра $p=m / N$ является интервал

$$
\left(\hat{p} \pm t_{\delta / 2} \sqrt{\varphi(\hat{p}) / m}\right)
$$


где $\hat{p}=m / \hat{N}$ и $\varphi(p)=p^{3} q^{s} /\left(1-q^{s}-s p q^{s-1}\right) ;$ для самого же параметра $N$ соответствующий доверительный интервал имеет вид

$$
\left(\hat{N} \pm t_{\delta / 2} \sqrt{\hat{N} \hat{q}^{s} /\left(1-\hat{q}^{s}-s \hat{p} \hat{q}^{s-1}\right)}\right)
$$

(подробнее об этом см. в п.6.1).

Дальнейшее обобщение результата (12) на случай произвольного ВП $\Pi_{n}\left(m_{1}, \ldots, m_{s}\right)$ получен в [34], где результат дан в следующей форме: если наблюдалось $\eta=l$, то

$$
\hat{N}(l)= \begin{cases}l & \text { при } l=m, \\ l+k_{l}-1 & \text { при } m<l<n, \\ \infty & \text { при } l=n,\end{cases}
$$

где

$$
k_{l}=\min \left\{k \geqslant 1: \prod_{i=1}^{s}\left(l+k-m_{i}\right)<k(l+k)^{s-1}\right\} .
$$

Заметим, что если ввести функцию

$$
S\left(N, r ; m_{1}, \ldots, m_{s}\right)=\left(\ln \frac{N+1}{N+1-r}\right) /\left(\frac{1}{s} \sum_{i=1}^{s} \ln \frac{N+1}{N+1-m_{i}}\right),
$$

где $m \leqslant r \leqslant N$, то результату (16) можно придать более традиционную форму типа (12), (14): при $m<\eta<n$ ОМП $\hat{N}$ находится из условия

$$
S\left(\hat{N}, \eta ; m_{1}, \ldots, m_{s}\right)<s \leqslant S\left(\hat{N}-1, \eta ; m_{1}, \ldots, m_{s}\right) .
$$

Более удобный для практических применений альтернативный алгоритм оценивания $N$ предложен в [24]: при $m<\eta<n$ ОМП $\hat{N}$ находится из условия

$$
\Phi_{s}(\hat{N}) \leqslant \eta<\Phi_{s}(\hat{N}+1)
$$

где (см. (3))

$$
\Phi_{s}(N)=\mathbf{E}_{N} \eta=N \Psi_{s}^{-1}(N) \Delta \Psi_{s}(N-1)=N\left(1-\prod_{i=1}^{s}\left(1-\frac{m_{i}}{N}\right)\right), \quad N \geqslant m
$$

если же $\eta=m$, то $\hat{N}=m$, а при $\eta=n$ оценка не определена $(\hat{N}=\infty)$. Другими словами, $\hat{N}=\max \left\{n \geqslant \eta: \Phi_{s}(N) \leqslant \eta\right\}=\Phi_{s}^{-1}(\eta)$, т.е. $\hat{N}$ фактически совпадает с оценкой по методу моментов. Преимущество этого алгоритма перед предыдущим (16) состоит в том, что последовательность $\left\{\Phi_{s}(N), N \geqslant m\right\}$ не зависит от значения наблюдаемой статистики $\eta$, и потому ее можно рассчитать заранее при заданных значениях параметров ВП $\Pi_{n}\left(m_{1}, \ldots, m_{s}\right)$. В $[4,34,37]$ можно найти дальнейшие ссылки на относящиеся к рассматриваемой проблематике публикации.

Подчеркнем, что во всех рассмотренных выше случаях на параметрическое множество $\mathcal{N}=\{N\}$ не накладывается никаких априорных ограчичений, кроме естественного ограничения снизу $N \geqslant m$, что имеет следствием появление 
с положительной вероятностью значения $\hat{N}=\infty$. Это легко объяснить следующими рассуждениями. Для ВП $\Pi_{n}\left(m_{1}, \ldots, m_{s}\right)$ носителем распределения достаточной статистики $\eta$ при заданном значении $N$ является множество (см. (2)) $\mathcal{L}_{m, n}(N)=\{m, m+1, \ldots, n \wedge N\}$, правдоподобие пропорционально величине

$$
\left(\begin{array}{c}
N \\
l
\end{array}\right) \Psi_{s}^{-1}(N) \mathbf{I}(N \geqslant l)
$$

при $\eta=l \in \mathcal{L}_{m, n}(N)$, где $\mathbf{I}(A)$ - индикатор события $A$, и если $N \geqslant n$, то для максимального значения статистики $\eta=n$ правдоподобие, как легко видеть, монотонно возрастает по $N$. Следовательно, если множество $\mathcal{N}$ априори не ограничено сверху, то формально мы должны положить $\hat{N}=\infty$. Таким образом, в этом случае ОМП $\hat{N}$ является смещенной, и ее математическое ожидание равно бесконечности. Для устранения этой особенности оценки $\hat{N}$ ее приходится тем или иным образом подправлять. Например, для указанной выше оценки Феллера известна модернизация вида

$$
\hat{N}^{\prime}=\frac{\left(m_{1}+1\right)\left(m_{2}+1\right)}{m_{1}+m_{2}+1-\eta}-1,
$$

которая оказывается несмещенной оценкой $N$ при $N \leqslant n$ (см. 55.4 в [1] и [25]). В то же время для моделей с априори ограниченным сверху множеством возможных значений $N$ подобной проблемы не возникает, и ОМП $\hat{N}$ в таких случаях всегда определена.

Другой заслуживающей быть отмеченной особенностью ОМП $\hat{N}$ в ОСВ является то, что при наблюдении минимального значения статистики $\eta=m$ оценка не зависит от числа выборок $s$, а определяется лишь априорным знанием того, что $N \geqslant m$. Это также легко следует из того, что в данном случае величина

$$
\left(\begin{array}{l}
N \\
m
\end{array}\right) \Psi_{s}^{-1}(N) \mathbf{I}(N \geqslant m)
$$

монотонно убывает по $N$.

\section{2. ОМП состава расслоенной совокупности}

Хорошо известно, что если элементы совокупности $U$ заданного размера $N^{0}$ классифицируются по альтернативному признаку, т.е. $U=U_{1} \cup U_{2}, N_{1}+N_{2}=N^{0}$, то ОМП размера $n<N^{0}$ имеет вид $\hat{N}_{1}=\left[\left(N^{0}+1\right) \eta_{1} / n\right]$, что легко выводится из свойств гипергеометрического распределения.

В общем случае произвольного ВП $\Pi_{n}\left(m_{1}, \ldots, m_{s}\right)$ и произвольного числа классов $k \geqslant 2$ при построении ОМП $\hat{N}=\left(\hat{N}_{1}, \ldots, \hat{N}_{k}\right)$ мы будем акцентировать внимание на двух наиболее интересных ситуациях, когда параметрическое множество модели имеет вид либо $\mathcal{N}=\mathcal{N}_{N^{0}}$ (см. п.2.1), либо $\mathcal{N}=\{\bar{N}: N \geqslant m\}$.

Случай $\mathcal{N}=\mathcal{N}_{N^{0}}, N^{0}>m$. В этом случае ОМП $\hat{N}=\left(\hat{N}_{1}, \ldots, \hat{N}_{k}\right)$ есть такая точка $k$-мерной гиперплоскости $\mathcal{N}_{N^{0}}=\left\{\bar{N}: N_{1}+\ldots+N_{k}=N^{0}\right\}$, в которой выполняется неравенство $L(\bar{N}+\bar{\delta} ; \bar{l}) \leqslant L(\bar{N} ; \bar{l})$. Здесь (см. (2))

$$
L(\bar{N} ; \bar{l})=\prod_{j=1}^{k}\left(\begin{array}{c}
N_{j} \\
l_{j}
\end{array}\right),
$$


$\bar{l}$ есть наблюденное значение статистики $\bar{\eta}$ и $\bar{\delta}=\left(\delta_{1}, \ldots, \delta_{k}\right)$ - произвольный ненулевой вектор с целочисленными координатами, удовлетворяющий условиям $\delta_{1}+\ldots+\delta_{k}=0$ и $N_{j}+\delta_{j} \geqslant l_{j} \forall j$. Положим $J_{-}=\left\{j: \delta_{j}<0\right\}, J_{+}=\left\{j: \delta_{j}>0\right\}$, тогда

$$
\begin{aligned}
\frac{L(\bar{N}+\bar{\delta} ; \bar{l})}{L(\bar{N} ; \bar{l})} & =\prod_{j \in J_{-}} \frac{\left(N_{j}+\delta_{j}+1-l_{j}\right) \ldots\left(N_{j}-l_{j}\right)}{\left(N_{j}+\delta_{j}+1\right) \ldots N_{j}} \prod_{j \in J_{+}} \frac{\left(N_{j}+1\right) \ldots\left(N_{j}+\delta_{j}\right)}{\left(N_{j}+1-l_{j}\right) \ldots\left(N_{j}+\delta_{j}-l_{j}\right)} \\
& =\prod_{j \in J_{-}}\left(1-\frac{l_{j}}{N_{j}+\delta_{j}+1}\right) \ldots\left(1-\frac{l_{j}}{N_{j}}\right) / \prod_{j \in J_{+}}\left(1-\frac{l_{j}}{N_{j}+1}\right) \ldots\left(1-\frac{l_{j}}{N_{j}+\delta_{j}}\right) \\
& \leqslant \prod_{j \in J_{-}}\left(1-\frac{l_{j}}{N_{j}}\right)^{\left|\delta_{j}\right|} / \prod_{j \in J_{+}}\left(1-\frac{l_{j}}{N_{j}+1}\right)^{\delta_{j}} .
\end{aligned}
$$

В частности, если все ненулевые $\delta_{j}= \pm 1$, то в последнем неравенстве имеет место знак равенства, и рассматриваемое отношение будет не больше единицы тогда и только тогда, когда выполняется условие

$$
\frac{l_{j}}{N_{j}+1} \leqslant \frac{l_{i}}{N_{i}} \quad \forall i \neq j .
$$

Так как, по условию,

$$
\sum_{j \in J_{-}}\left|\delta_{j}\right|=\sum_{j \in J_{+}} \delta_{j},
$$

точка $\bar{N}$, удовлетворяющая (18), будет одновременно точкой глобального максимума функции $L(\bar{N} ; \bar{l})$. Тем самым, любое решение неравенств $(18)$, принадлежащее гиперплоскости $\mathcal{N}_{N^{0}}$, является ОМП $\hat{N}$.

Отметим некоторне свойства решений системы (18), проясняющие их характер. Суммируя неравенства $l_{j} N_{i} \leqslant l_{i}\left(N_{j}+1\right), i \neq j$, при фиксированном $j$, получим неравенство $l_{j}\left(N^{0}-N_{j}\right) \leqslant\left(l-l_{j}\right)\left(N_{j}+1\right)$ (здесь и далее $l=l_{1}+\ldots+l_{k}$ ) или $l_{j}\left(N^{0}+1\right) \leqslant l\left(N_{j}+1\right)$. Таким образом, $l_{j} /\left(N_{j}+1\right) \leqslant l /\left(N^{0}+1\right)$ для всех $j=1, \ldots, k$. С другой стороны, суммируя те же неравенства по $j$ при фиксированном $i$, мы получим, что $\left(l-{ }_{i}\right) N_{i} \leqslant l_{i}\left(N^{0}-N_{i}+k-1\right)$, т.e. $l_{i} / N \geqslant l /\left(N^{0}+k-1\right)$ для всех $i=1, \ldots, k$.

При $k=2$ полученные верхняя и нижняя границы совпадают, следовательно, система (18) в этом случае эквивалентна системе

$$
\frac{l_{j}}{N_{j}+1} \leqslant \frac{l}{N^{0}+1} \leqslant \frac{l_{i}}{N_{i}}, \quad i \neq j,
$$

которая определяет ОМП $\hat{N}=\left(\hat{N}_{1}, \hat{N}_{2}\right)$ в явной форме

$$
\left(N^{0}+1\right) l_{j} / l-1 \leqslant \hat{N}_{j} \leqslant\left(N^{0}+1\right) l_{j} / l, \quad j=1,2
$$

На этом же пути можно построить простые решения системы (18) и при любом $k>2$. Именно, возьмем произвольное $c \in[1, k-1]$ и рассмотрим систему неравенств

$$
\frac{l_{j}}{N_{j}+1} \leqslant \frac{l}{N^{0}+\mathrm{c}} \leqslant \frac{l_{i}}{N_{i}}, \quad i \neq j .
$$


Ясно, что решение этой системы является одновременно решением и системы (18), и для него мы имеем явный вид

$$
\left(N^{0}+c\right) l_{j} / l-1 \leqslant \hat{N}_{j} \leqslant\left(N^{0}+c\right) l_{j} / l, \quad j=1, \ldots, k
$$

Так как нам нужны лишь решения, удовлетворяющие условию $\hat{N}_{1}+\ldots+\hat{N}_{k}=N^{0}$, свободный параметр $c$ должен быть выбран так, чтобы обеспечить это равенство. Однако вместо этого можно предложить несколько модифицированную версию ОМП, удобную для практического использования, именно,

$$
\hat{N}_{j}=\left[\left(N^{0}+1\right) l_{j} / l\right], \quad j=1, \ldots, k-1, \quad \hat{N}_{k}=N^{0}-\sum_{j=1}^{k-1} \hat{N}_{j}
$$

(для случая двух классов $(k=2)$ эта оценка совпадает с точным решением). В итоге мы имеем следующий результат.

Теорема 5. При известном размере $N^{0}$ совокупности $U=\bigcup_{j=1}^{k} U_{j}$ ОМП ее состава $\bar{N}=\left(N_{1}, \ldots, N_{k}\right)$ в ОСВ определяется системой неравенств (18), а ее модифицированная версия дается формулами (19).

Сделаем несколько дополнительных замечаний к этому результату. Прежде всего, из предыдущего следует, что ОМП $\hat{N}$, вообще говоря, определяется неоднозначно (факт, вообще типичный для моделей с дискретным параметром). Далее, ОМП $\hat{N}_{j}$ для размера $j$-го слоя приблизительно пропорциональна наблюденному числу $l_{j}$ различных элементов этого слоя; грубо говоря, $\tilde{N}_{j} \approx N^{0} l_{j} / l$, $j=1, \ldots, k$, и ошибка этой аппроксимации незначительна при больших $N^{0}$. Наконец, так как (см. (3) и (6))

$$
\mathbf{E}_{\bar{N}} \eta_{j}=N_{j} \Psi_{s}^{-1}\left(N^{0}\right) \Delta \Psi_{s}\left(N^{0}-1\right)=N_{j} \mathbf{E}_{\bar{N}} \eta / N^{0},
$$

справедливо равенство

$$
N_{j}=N^{0} \mathbf{E}_{\bar{N}} \eta_{j} / \mathbf{E}_{\bar{N}} \eta
$$

Заменяя здесь теоретические средние соответствующими наблюденными значениями, мы получаем оценки по методу моментов: $\tilde{N}_{j}=N^{0} l_{j} / l, j=1, \ldots, k$. Таким образом, ОМП $\hat{N}_{j}$ практически совпадает с оценками $\tilde{N}_{j}$ метода моментов.

Рассмотрим теперь случай $\mathcal{N}=\{\bar{N}: N \geqslant m\}$. Здесь неизвестным параметром является также и общий размер совокупности $N$, который может быть оценен предварительно по объединенным данным $\eta_{1}+\ldots+\eta_{k}$ с использованием приведенных выше алгоритмов (16) или (17). Определенная на первом этапе ОМП $\hat{N}$ общего размера совокупности задает гиперплоскость $\mathcal{N}_{\hat{N}}=\left\{\bar{N}: N_{1}+\ldots+N_{k}=\right.$ $\hat{N}\}$, на которой лежит искомая точка $\left(\hat{N}_{1}, \ldots, \hat{N}_{k}\right)$, что по существу сводит задачу построения ОМП для размеров слоев совокупности $U$ к рассмотренному выше случаю с $N^{0}=\hat{N}$, т.е. мы приходим к оценкам (см. (19))

$$
\hat{N}_{j}=\left[(\hat{N}+1) \eta_{j} / \eta\right], \quad j=1, \ldots, k-1, \quad \hat{N}_{k}=\hat{N}-\sum_{j=1}^{k-1} \hat{N}_{j}
$$


Таким образом, в данном случае ОМП состава $\bar{N}=\left(N_{1}, \ldots, N_{k}\right)$ совокупности $U=\bigcup_{j=1}^{k} U_{j}$ строится в два этапа с использованием соответствующих результатов для нерасслоенной совокупности (алгоритм (17)) и оценок вида (19) для расслоенной совокупности с известным общим размером.

Замечание 1. Как уже отмечалось выше, при наблюдении $\eta=n$ (что возможно лишь при $N \geqslant n$ ) принцип правдоподобия приводит к оценке $\hat{N}=\infty$, если априорное ограничение сверху на общий размер совокупности отсутстзует. Как избежать этой неприятности для оценки Феллера в двухвыборочном случае $(s=2)$, сказано выше. Для произвольной ОСВ по аналогии можно предложить, например, следующую модернизацию ОМП: в (17) $\eta$ заменить на $\eta \wedge(n-1)$, что формально можно записать в виде

$$
\hat{N}^{\prime}=\Phi_{s}^{-1}(\eta) \mathbf{I}(\eta<n)+\Phi_{s}^{-1}(n-1) \mathbf{I}(\eta=n) .
$$

Замечание 2. В тех случаях, когда с вероятностью 1 ОМП $\hat{N}$ конечна (например, когда априори $N \leqslant M<\infty$, см. $\mathcal{N}^{(3)}$ в п.2.1), из (17) можно получить также некоторую информацию о ее смещении. Именно, принимая во внимание, что $\mathbf{E}_{\bar{N}} \eta=\Phi_{s}(N)$, а также выпуклость вверх функции $\Phi_{s}(N)$, по неравенству Иенсена из (17) имеем $\mathbf{E}_{\bar{N}} \eta=\Phi_{s}(N)<\mathbf{E}_{\bar{N}} \Phi_{s}(\hat{N}+1) \leqslant \Phi_{s}\left(\mathbf{E}_{\bar{N}} \hat{N}+1\right)$, т.e. $\mathbf{E}_{\bar{N}} \hat{N} \geqslant N-1$.

\section{5. Оценивание для больших выборок}

В этом разделе мы приводим результаты для случая больших выборок (т.е. при $n \rightarrow \infty)$, предполагая априори, что общий размер совокупности и размеры составляющих ее классов также велики (некоторые асимптотические результаты для нерасслоенных совокупностей приведены выше в п.4.1).

Поскольку анализируемая модель имеет много степеней свободы (многомерный оцениваемый параметр $\bar{N}=\left(N_{1}, \ldots, N_{k}\right)$, произвольные параметры $\left.m_{1}, \ldots, m_{s} \mathrm{OCB}\right)$, то содержательные результаты для нее получаются лишь при соответствующих условиях регулярности на определяющие ее параметры. В качестве примеров таковых условий на параметры ОСВ мы будем выделять далее два следующих варианта, представляющих, на наш взгляд, наибольший интерес для приложений:

- $m \geqslant 1$ фиксировано, а $s \rightarrow \infty$, так что $n=m_{1}+\ldots+m_{s} \rightarrow \infty$;

$-s \geqslant 2$ фиксировано, а $m_{i} \rightarrow \infty$ так, что $\lim m_{i} / n=\rho_{i} \in[\delta, 1-\delta], \delta>0$, $i=1, \ldots, s$.

Что касается априорных предположений о структуре совокупности $U$, то будем считать, что число классов $k$ фиксировано и известно, а оцениваемые параметры $\bar{N}$ удовлетворяют соотношениям

$N_{j}=\beta_{j} N, \quad \beta_{j} \in\left[\delta_{1}, 1-\delta_{1}\right], \quad \delta_{1}>0, \quad j=1, \ldots, k, \quad 0<\alpha_{1} \leqslant \alpha=n / N \leqslant \alpha_{2}<\infty$, 
так что $N$ и все $N_{j}, j=1, \ldots, k$, имеют порядки, сравнимые с общим числом наблюдений $n$.

При асимптотическом подходе естественно рассматривать в качестве оцениваемых параметров вектор долей $\bar{\beta}=\left(\beta_{1}, \ldots, \beta_{k}\right)$ и величину $\alpha=n / N$ (когда $N$ неизвестно), которые полностью определяют структуру исследуемой совокупности $U$, и ниже мы строим асимптотические ОМП для этих параметров, которые при выполнении описанных выше условий обладают свойствами состоятельности и асимптотической нормальности (с.а.н). Заметим также, что эти условия являются типичными для теории больших выборок в подобного рода задачах, и именно такие условия позволяют построить достаточно полную (можно сказать регулярную) асимптотическую теорию соответствующих статистических выводов $[6,16,33]$.

\section{1. Состоятельные асимптотически нормальные оценки}

Нетрудно убедиться в том, что при сформулированных условиях функция $\Phi_{s}(N)$ в (17) удовлетворяет асимптотическому соотношению

$$
\Phi_{s}(N) / n=G(\alpha)+O(1 / n)
$$

при этом функция $G(\alpha)$ монотонно убывает по $\alpha$, следовательно, определена обратная к ней функция $G^{-1}(t)$. В первом выделенном выше случае $G(\alpha)=$ $(1-\exp (-\alpha)) / \alpha$, а во втором $G(\alpha)=\left(1-\prod_{i=1}^{s}\left(1-\alpha \rho_{i}\right)\right) / \alpha$.

После этих предварительных замечаний перейдем к задаче оценивания неизвестных параметров рассматриваемой модели. Предположим, что при наблюденных данных $\bar{\eta}=\left(\eta_{1}, \ldots, \eta_{k}\right)$ осуществляется событие

$$
A_{n}=\left\{\eta / n \in[\varepsilon, 1-\varepsilon] \exists \varepsilon>0, \eta_{j} / \eta \in\left[\varepsilon_{1}, 1-\varepsilon\right] \exists \varepsilon_{1}>0 \forall j\right\} .
$$

Как следует из [16, Теорема 2], где детально исследовано асимптотическое поведение распределения статистики $\eta$ в рассматриваемых условиях, $\mathbf{P}\left(A_{n}\right) \rightarrow 1$ при $n \rightarrow \infty$.

Если осуществляется событие $A_{n}$, то из (22) следует, что условие (17) асимптотически эквивалентно условию $G\left(\hat{\alpha}_{n}\right)=\eta / n, \hat{\alpha}_{n}=n / \hat{N}$. Отсюда получаем, что асимптотической ОМП для параметра $\alpha$ (когда $N$ неизвестно) является статистика

$$
\hat{\alpha}_{n}=G^{-1}(\eta / n) .
$$

В свою очередь, из (19) и (20) следует, что, как в случае известного, так и в случае неизвестного общего размера совокупности, асимптотической ОМП для вектора долей $\bar{\beta}=\left(\beta_{1}, \ldots, \beta_{k}\right)$ является статистика

$$
\hat{\beta}_{n}=\left(\hat{\beta}_{1 n}, \ldots, \hat{\beta}_{k n}\right)=\bar{\eta} / \eta
$$

Соотношения (23) и (24) полностью решают задачу асимптотического точечного оценивания параметров модели на основании принципа правдоподобия.

Замечание 3. Из (23) можно также заключить, что асимптотическая ОМП для $N$ имеет вид $\hat{N}=n / G^{-1}(\eta / n)$. В этой связи отметим, что при $n \geqslant N$ существует 
НОМД для $N$ (см. теорему 2), именно, $N^{*}=\Delta^{\eta}\left(0 \Psi_{s}(0)\right) / \Delta^{\eta} \Psi_{s}(0)$. Воспользовавшись известной формулой Гуда [29] для чисел Моргана $\Delta^{l} 0^{n}:$ при $l, n \rightarrow \infty$ и $0<c_{1} \leqslant \nu=l / n \leqslant c_{2}<1$

$$
\Delta^{l} 0^{n}=\frac{n !(\exp (\lambda)-1)^{l}}{\lambda^{n} \sqrt{2 \pi n\left(1+\lambda-\nu^{-1}\right)}}\left(1+O\left(\frac{1}{n}\right)\right),
$$

где $\lambda-$ корень уравнения $(1-\exp (-\lambda)) / \lambda=\nu$. Из этой оценки следует, что для ограниченных по модулю $j= \pm 1, \pm 2, \ldots$

$$
\frac{\Delta^{l} 0^{n+j}}{\Delta^{l} 0^{n}}=\left(\frac{n}{\lambda}\right)^{j}\left(1+O\left(\frac{1}{n}\right)\right) \text {. }
$$

Нетрудно показать, что при $n \geqslant N$ в первом выделенном выше случае $\hat{N} \sim N^{*}$. Этот факт для схемы простой повторной выборки $\Pi_{n}$ отмечен в [32]. Такое же заключение кажется правдоподобным и во втором случае.

Асимптотические распределения оценок (23) и (24), позволяющие рассчитывать соответствующие асимптотические доверительные интервалы и области, получены в [16]. Чтобы сформулировать эти результаты, положим

$$
a_{N}=\Phi_{s}(N) / N, \quad a=\lim _{N \rightarrow \infty} a_{N}=a(\alpha)=\alpha G(\alpha)
$$

где $G(\alpha)$ определено в $(22)$, и

$$
b(\alpha)=\lim _{N \rightarrow \infty}\left(1-a_{N}\right)\left(a_{N}-(N-1)\left(a_{N-1}-a_{N}\right)\right) .
$$

Отметим также, что в первом случае

$$
b(\alpha)=(1-a)(1-(1-a)(1+\alpha)),
$$

а во втором

$$
b(\alpha)=(1-a)\left(1-(1-a)\left(1+\alpha \sum_{i=1}^{s} \frac{\rho_{i}}{1-\alpha \rho_{i}}\right)\right)
$$

Теорема 6. При выполнении условий (21) статистики $\hat{\alpha}_{n}=G^{-1}(\eta / n) u \hat{\beta}_{n}=\bar{\eta} / \eta$ являются с.а.н. оченками $\alpha$ и $\bar{\beta}$ соответственно, при этом $\mathcal{L}\left(\hat{\alpha}_{n}\right) \sim \mathcal{N}\left(\alpha, \sigma^{2}(\alpha) / n\right)$ $u \mathcal{L}\left(\hat{\beta}_{n}\right) \sim \mathcal{N}(\bar{\beta}, \Sigma(\alpha, \bar{\beta}) / n)$, дде

$$
\sigma^{2}(\alpha)=\frac{b(\alpha)}{\alpha\left(G^{\prime}(\alpha)\right)^{2}}, \quad \Sigma(\alpha, \bar{\beta})=\psi(\alpha)\left\|\beta_{i}\left(\delta_{i j}-\beta_{j}\right)\right\|_{1}^{k}, \quad \psi(\alpha)=\frac{1-\alpha G(\alpha)}{G(\alpha)}
$$

$\delta_{i j}$ - символ Кронекера.

Более того, в этих асимптотических соотношениях в выражениях для вторых моментов неизвестные параметры $\alpha$ и $\bar{\beta}$ могут быть заменены их оценками $\hat{\alpha}_{n}$ $u \hat{\beta}_{n}$. 


\section{2. Асимптотические доверительные интервалы и области}

Стандартная техника использования теоремы 6 для построения доверительных интервалов и областей приводит к следующим результатам: асимптотические $(1-\delta)$-доверительные интервалы для $\alpha$ и $\beta_{j}$ имеют вид соответственно

$$
\left(\hat{\alpha}_{n} \pm t_{\delta / 2} \sigma\left(\hat{\alpha}_{n}\right) / \sqrt{n}\right)
$$

и

$$
\left(\hat{\beta}_{j n} \pm t_{\delta / 2} \sqrt{\psi\left(\hat{\alpha}_{n}\right) \hat{\beta}_{j n}\left(1-\hat{\beta}_{j n}\right) / n}\right)
$$

а асимптотическое $\gamma$-доверительное множество для вектора $\bar{\beta}$ имеет вид

$$
\mathcal{G}_{\gamma_{n}}=\left\{\bar{\beta}: \sum_{j=1}^{k} \frac{\left(\hat{\beta}_{j n}-\beta_{j}\right)^{2}}{\hat{\beta}_{j n}}<\frac{\chi_{\gamma, k-1}^{2} \psi\left(\hat{\alpha}_{n}\right)}{n}, \quad \sum_{j=1}^{k} \beta_{j}=1\right\},
$$

где $\chi_{\gamma, k-1}^{2}$ есть $\gamma$-квантиль распределения $\chi^{2}$ с $k-1$ степенями свободы.

Множества (25) можно переписать в виде

$$
\mathcal{G}_{\gamma n}=\left\{\hat{\beta}^{0}:\left|\bar{\lambda}^{\prime}\left(\hat{\beta}_{n}^{0}-\bar{\beta}^{0}\right)\right|<\sqrt{\chi_{\gamma, k-1}^{2} \psi\left(\hat{\alpha}_{n}\right)\left(\bar{\lambda}^{\prime} H_{n} \bar{\lambda}\right) / n} \quad \forall \bar{\lambda}\right\},
$$

где

$$
\bar{\beta}^{0}=\left(\beta_{1}, \ldots, \beta_{k-1}\right), \quad H_{n}=H\left(\hat{\beta}_{n}^{0}\right), \quad H\left(\bar{\beta}^{0}\right)=\left\|\beta_{i}\left(\delta_{i j}-\beta_{j}\right)\right\|_{1}^{k-1} .
$$

Отсюда следует, что для всех линейных комбинаций $\left\{\bar{\lambda}^{\prime} \bar{\beta}^{0}\right\}$ система доверительных интервалов

$$
\left\{\left(\bar{\lambda}^{\prime} \hat{\beta}_{n}^{0} \pm \sqrt{\chi_{\gamma, k-1}^{2} \psi\left(\hat{\alpha}_{n}\right)\left(\bar{\lambda}^{\prime} H_{n} \bar{\lambda}\right) / n}\right)\right\}
$$

обладает асимптотически (в условиях теоремы 6) доверительным уровнем $\gamma$. Другими словами, (26) есть система совместных асимптотических доверительных интервалов для произвольных линейных комбинаций $\left\{\bar{\lambda}^{\prime} \bar{\beta}^{0}\right\}$. Если требуется одновременно оценить конечное число таких комбинаций, то система соответствующих этим комбинациям доверительных интервалов из (26) будет обладать асимптотически доверительным уровнем, не меньшим $\gamma$. Для сравнения приведем вид асимптотического $(1-\delta)$-доверительного интервала для отдельной заданной линейной комбинации $\bar{\lambda}^{\prime} \bar{\beta}^{0}=\lambda_{1} \beta_{1}+\ldots+\lambda_{k-1} \beta_{k-1}$ :

$$
\left(\bar{\lambda}^{\prime} \hat{\beta}_{n}^{0} \pm t_{\delta / 2} \sqrt{\psi\left(\hat{\alpha}_{n}\right)\left(\bar{\lambda}^{\prime} H_{n} \bar{\lambda}\right) / n}\right) .
$$

В частности, из предыдущего следует, что система совместных доверительных интервалов для самих параметров $\left\{\beta_{j}\right\}$ (всех или части) имеет вид

$$
\left\{\left(\hat{\beta}_{j n} \pm \sqrt{\chi_{\gamma, k-1}^{2} \psi\left(\hat{\alpha}_{n}\right) \hat{\beta}_{j n}\left(1-\hat{\beta}_{j n}\right) / n}\right)\right\}
$$

их доверительный уровень асимптотически не меньше $\gamma$; аналогичная же система для разностей $\left\{\beta_{i}-\beta_{j}, j<i\right\}$ имеет вид

$$
\left\{\left(\hat{\beta}_{i n}-\hat{\beta}_{j n} \pm \sqrt{\chi_{\gamma, k-1}^{2} \psi\left(\hat{\alpha}_{n}\right)\left(\hat{\beta}_{i n}+\hat{\beta}_{j n}-\left(\hat{\beta}_{i n}-\hat{\beta}_{j n}\right)^{2}\right) / n}\right), \quad j<i\right\} .
$$


Заметим, что теорема 6 позволяет строить с.а.н. ОМП $\hat{\tau}_{n}=\tau\left(\hat{\alpha}_{n}, \hat{\beta}_{n}\right)$ для произвольных гладких функций $\tau(\alpha, \bar{\beta})$ и рассчитывать для них асимптотические доверительные интервалы.

Замечание 4. После того, как данный обзор был уже написан, нам стала известна работа [43], в которой метод максимального правдоподобия применен для оценивания состава расслоенной совокупности (ее общий размер также может быть неизвестен) в схеме неравновероятной бесповторной выборки, когда выбор элементов производится последовательно с вероятностями, пропорциональными некоторым (неизвестным) весам, характеризующим емкость элементов совокупности. Именно, если $S_{n}=\left(U_{i_{1}}, \ldots, U_{i_{n}}\right)$ есть упорядоченная выборка объема $n$ элементов совокупности, то вероятность ее извлечения имеет вид

$$
\mathbf{P}\left(S_{n}\right)=\prod_{j=1}^{n} w_{i j}\left(\sum_{i=1}^{N} w_{i}-\sum_{\nu=1}^{j-1} w_{i_{\nu}}\right)^{-1}
$$

где $w_{j}, j=1, \ldots, N$, - веса соответствующих элементов. В этой работе при общих предположениях о модели получены асимптотически (для больших выборок) эффективные аппроксимации для ОМП неизвестного состава совокупности, методом статистического моделирования проведено исследование этих оценок для малых выборок и результаты применены к анализу геологических данных о месторождениях нефти и газа в бассейне Северного моря. Также в работе имеется обширная библиография по модели с неравновероятным бесповторным выбором.

\section{6. Разные задачи}

В этом разделе мы приводим отдельные результаты в обсуждаемой проблематике. Некоторые из этих результатов, вообще говоря, можно рассматривать в качестве начальных для более систематических исследований.

\section{1. Сравнение выборочных планов}

При выборочном обследовании конечных совокупностей ключевым является вопрос организации стохастического эксперимента для получения исходной статистической информации. В тех случаях, когда выбор плана обследования зависит от исследователя (активный эксперимент), возникает проблема сравнения различных способов получения выборки и определения наилучшего (в том или ином смысле) из них. Решение подобных задач зависит, с одной стороны, от структуры исследуемой совокупности $U$ и связанной с этим цели исследования и, с другой стороны, от типа сравниваемых выборочных планов и принимаемого критерия сравнения.

В качестве иллюстративного примера рассмотрим задачу оценивания неизвестного параметра $N$ однородной совокупности $U$, когда в качестве конкурирующих вариантов ВП при заданном общем ресурсе испытаний $n$ выступают либо план $\Pi_{n}(s, m)$, состоящий в независимом и равновероятном извлечении из 
совокупности $s$ раз по $m$ элементов $(n=s m)$, либо план простой повторной выборки $\Pi_{n}=\Pi_{n}(n, 1)$. Как следует из п.3.2, для произвольного $\mathrm{B} \Pi \Pi_{n}(s, m)$ при $N \leqslant n$ для $N$ существует НОМД

$$
N_{s, m}^{*}=\frac{\Delta^{\eta}\left(0 \Psi_{s}(0)\right)}{\Delta^{\eta} \Psi_{s}(0)}, \quad \Psi_{s}(N)=\left(\begin{array}{c}
N \\
m
\end{array}\right)^{s},
$$

если же априорные ограничения сверху на размер совокупности отсутствуют, то НОМД можно построить для обратной величины $N^{-1}$ :

$$
\left(\frac{1}{N}\right)_{s, m}^{*}=\frac{\Delta^{\eta}\left(0^{-1} \Psi_{s}(0)\right)}{\Delta^{\eta} \Psi_{s}(0)}
$$

Для плана $\Pi_{n}$ оценки (27), (28) принимают соответственно вид

$$
N_{n}^{*}=\frac{\Delta^{\eta} 0^{n+1}}{\Delta^{\eta} 0^{n}}, \quad\left(\frac{1}{N}\right)_{n}^{*}=\frac{\Delta^{\eta} 0^{n-1}}{\Delta^{\eta} 0^{n}}
$$

Вычислить дисперсии $\mathbf{D} N^{*}$ и $\mathbf{D}\left(N^{-1}\right)^{*}$ этих оценок и провести их сравнение в точном виде весьма затруднительно, поэтому можно, например, сравнить соответствующие несмещенные оценки этих дисперсий, которые имеют вид

$$
\mathbf{D}^{*} N^{*}=\left(N^{*}\right)^{2}-\left(N^{2}\right)^{*}, \quad \mathbf{D}^{*}\left(\frac{1}{N}\right)^{*}=\left(\left(\frac{1}{N}\right) *\right)^{2}-\left(\frac{1}{N^{2}}\right)^{*},
$$

где несмещенные оценки степеней $N^{ \pm 2}$ вычисляются по формулам, аналогичным (27), (28):

$$
\left(N^{ \pm 2}\right)_{s, m}^{*}=\Delta^{\eta}\left(0^{ \pm 2} \Psi_{s}(0)\right) / \Delta^{\eta} \Psi_{s}(0)
$$

Проблема практических вычислений по этим формулам обсуждется в [26], см. также [3, с.238].

Однако можно решить эту задачу в асимптотической постановке, предполагая размер $N$ совокупности и общее число наблюдений $n$ большими. Именно, будем предполагать, что $m, N \rightarrow \infty$ так, что

$$
p=\frac{m}{N}=1-q \in\left[p_{1}, p_{2}\right], \quad 0<p_{1}<p_{2}<1
$$

При этом условии величину $s$ (число выборок) будем считать фиксированной . $(s \geqslant 2)$; положим также $\alpha=n / N$, следовательно, $\alpha=s p$.

Как показано в [13], при условии (30) существует асимптотически оптимальная (в некотором естественном классе оценок) оценка $\hat{p}_{s, m}$ для параметра $p$; эта оценка имеет вид

$$
\hat{p}_{s, m}=b_{s}^{-1}(\eta / m)
$$

где $b_{s}^{-1}(t)-$ функция, обратная к $b_{s}(p)=\left(1-q^{s}\right) / p$, и при этом

$$
\mathcal{L}\left(\hat{p}_{s, m}\right) \sim \mathcal{N}\left(p, \varphi_{1, s}(p) / m\right),
$$

где $\varphi_{1, s}(p)=p^{3} q^{s}\left(1-q^{s}-s p q^{s-1}\right)^{-1}$. Таким образом, $\hat{p}_{s, m}$ является с.а.н. оценкой $p$. 
Аналогичный результат для плана $\Pi_{n}$ формулируется в терминах параметра $\alpha$ следующим образом [14]: с.а.н. оценка $\alpha$ имеет вид

$$
\hat{\alpha}_{n}=a^{-1}(\eta / n)
$$

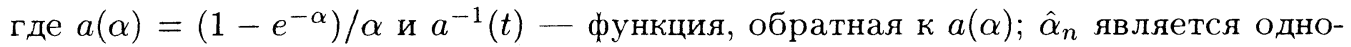
временно асимптотической ОМП для $\alpha$ и при этом

$$
\mathcal{L}\left(\hat{\alpha}_{n}\right) \sim \mathcal{N}\left(\alpha, \varphi_{2}(\alpha) / n\right)
$$

где $\varphi_{2}(\alpha)=\alpha^{3}\left(e^{\alpha}-1-\alpha\right)^{-1}$.

Әти результаты позволяют предложить следующие собственные оценки для самого параметра $N$ : для плана $\Pi_{n}(s, m)$ асимптотическим вариантом оценки $N$ является статистика (см. (31)) $\hat{N}_{s, m}=m / \hat{p}_{s, m}$, а для плана $\Pi_{n}$ статистика (см. (33)) $\hat{N}_{n}=n / \hat{\alpha}_{n}$. При этом из (32) следует, что в условиях (30)

$$
\mathcal{L}\left(\hat{N}_{s, m}\right) \sim \mathcal{N}\left(N, N \varphi_{1, s}(p) / p^{3}\right)
$$

а из (34) следует, что

$$
\mathcal{L}\left(\hat{N}_{n}\right) \sim \mathcal{N}\left(N, N \varphi_{2}(\alpha) / \alpha^{3}\right)
$$

(из этих соотношений, в частности, получаются соответствующие асимптотические доверительные интервалы для $N$, см. (13) и (15)).

Соотношения $(35),(36)$ и являются исходными для решения задачи асимптотического сравнения планов $\Pi_{n}(s, m)$ и $\Pi_{n}$ в условиях (30): асимптотическую эффективность плана $\Pi_{n}$ по отношению к плану $\Pi_{n}(s, m)$ будем измерять отношением асимптотических дисперсий в (35) и (36), т.е. величиной

$$
e_{s}(p)=\frac{\varphi_{1, s}(p)}{p^{3}}:\left.\frac{\varphi_{2}(\alpha)}{\alpha^{3}}\right|_{\alpha=s p}=\frac{q^{s}\left(e^{s p}-1-s p\right)}{1-q^{s}-s p q^{s-1}}
$$

Если $e_{s}(p)<1$, то план $\Pi_{n}$ менее эффективен в условиях (30), в таком случае будем писать $\Pi_{n} \prec \Pi_{n}(s, m)$; если же $e_{s}(p)>1$, то $\Pi_{n} \succ \Pi_{n}(s, m)$.

Теорема 7. В условиях (30) справедливы соотношения $\Pi_{n} \prec \Pi_{n}(s, m)$ nри $p>p_{s}$ $u \Pi_{n} \succ \Pi_{n}(s, m)$ nри $p<p_{s}$, где $p_{s} \in(0,1)$ - единственный корень уравнения $e_{s}(p)=1$.

Доказательство следует из факта монотонного убывания функции $e_{s}(p)$ (см. (37)) от $e_{s}(0)=s /(s-1)$ до $e_{s}(1)=0$. 
Таким образом, в данном случае нет однозначного ответа, какой из двух планов, $\Pi_{n}$ или $\Pi_{n}(s, m)$, эффективнее: при малых значениях параметра $\left.p<p_{s}\right)$ более эффективным является план $\Pi_{n}$, а при значениях $p>p_{s}$ план $\Pi_{n}(s, m)$. Ниже приводится таблица этих критических значений $p_{s}$, а также краткая таблица значений функции эффективности $e_{s}(p)$ для $s=2, \ldots, 5$ :

\begin{tabular}{l|lllllllll}
$s$ & 2 & 3 & 4 & 5 & 6 & 7 & 8 & 9 & 10 \\
\hline$p_{s}$ & 0.3828 & 0.2456 & 0.18111 & 0,1435 & 0.1188 & 0.1014 & 0.0885 & 0.0785 & 0.0705
\end{tabular}

Также приведем таблицу значений функции $e_{s}(p)$ :

\begin{tabular}{l|lllllllll}
$s / p$ & 0.1 & 0.2 & 0.3 & 0.4 & 0.5 & 0.6 & 0.7 & 0.8 & 0.9 \\
\hline 2 & 1.73 & 1.47 & 1.21 & 0.96 & 0.72 & 0.50 & 0.30 & 0.15 & 0.04 \\
3 & 1.30 & 1.09 & 0.89 & 0.69 & 0.50 & 0.32 & 0.17 & 0.07 & 0.01 \\
4 & 1.15 & 0.96 & 0.77 & 0.58 & 0.40 & 0.24 & 0.11 & 0.03 & 0.00 \\
5 & 1.08 & 0.90 & 0.71 & 0.51 & 0.33 & 0.18 & 0.07 & 0.02 & 0.00
\end{tabular}

Еще один пример сравнения выборочных планов имеется в работе [23], где показано, что в задаче оценивания состава $\bar{N}=\left(N_{1}, \ldots, N_{k}\right) k$-слойной совокупности с известным общим размером $N^{0}=N_{1}+\ldots+N_{k}$ среди всех планов $\Pi_{n}\left(m_{1}, \ldots, m_{s}\right)$ с $n=m_{1}+\ldots+m_{s} \leqslant N$ наиболее эффективным является план простой бесповторной выборки $\bar{\Pi}_{n}$; критерием сравнения при этом является величина дисперсии линейных несмещенных оценок параметров $N_{j}$ (см. (11)).

\section{2. Проверка гипотез}

Проверка гипотез о составе исследуемой совокупности представляет собой, в общем, фактически не разработанную область теории; соответствующие результаты имеются здесь лишь для отдельных частных случаев. Так, в работе [12] для выборочных планов $\Pi_{n}(s, m)$ и $\Pi_{n}$ предложены асимптотически (в условиях (30)) несмещенные критерии проверки простых и сложных гипотез о параметрах $p$ и $\alpha$ соответственно и вычислены их мощности при близких альтернативах. Несколько усовершенствованный вариант этих результатов для плана $\Pi_{n}(s, m)$ формулируется следующим образом: при заданном уровне значимости $\delta$ критическая область для простой гипотезы $H_{0}: p=p_{0}, 0<p_{0}<1$, асимптотически имеет вид (см. (31), (32))

$$
S_{\delta}\left(p_{0}\right)=\left\{\left|\hat{p}_{s, m}-p_{0}\right| \sqrt{m / \varphi_{1, s}\left(p_{0}\right)}>t_{\delta / 2}\right\}
$$

для проверки же сложной гипотезы $H_{0}: p \in\left[p_{1}, p_{1}\right], 0<p_{1}<p_{2}<1$, соответствующая критическая область задается в виде

$$
S_{\delta}\left(p_{1}, p_{2}\right)=\left\{\hat{p}_{s, m}<p_{1}-t_{\delta} \sqrt{\varphi_{1, s}\left(p_{1}\right) / m}\right\} \cup\left\{\hat{p}_{s, m}>p_{2}+t_{\delta} \sqrt{\varphi_{1, s}\left(p_{2}\right) / m}\right\} .
$$

Эти критерии позволяют различать альтернативы, сближающиеся с гипотезой $H_{0}$ со скоростью $1 / \sqrt{m}$. Так, для случая простой гипотезы мощность критерия (38) при альтернативах вида $p=p(m)=p_{0}+b / \sqrt{m}$, где $b$ - некоторая ненулевая постоянная, асимптотически равна величине

$$
\Phi\left(b / \varphi_{1, s}\left(p_{0}\right)-t_{\delta / 2}\right)+\Phi\left(-b / \varphi_{1, s}\left(p_{0}\right)-t_{\delta / 2}\right)
$$




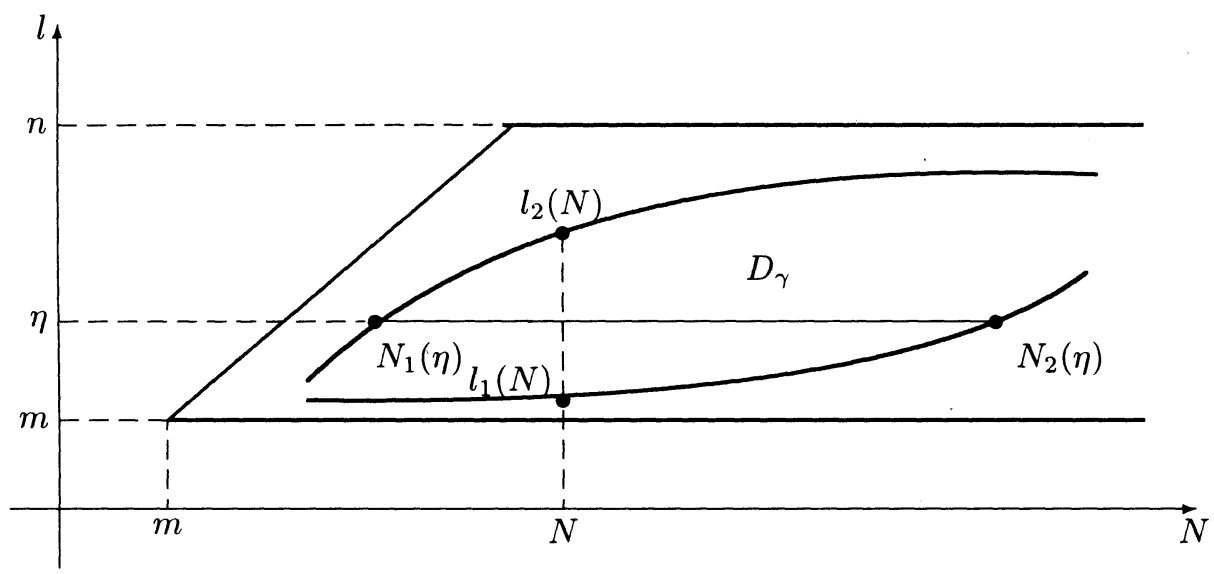

Рис. 1.

где $\Phi(x)$ - функция стандартного нормального распределения.

Для критерия (39) функция мощности $W_{m}(p)$ удовлетворяет следующим асимптотическим соотношениям:

- если $p_{1}<p<p_{2}$, и при этом $\min \left\{\sqrt{m}\left(p-p_{1}\right), \sqrt{m}\left(p_{2}-p\right)\right\} \rightarrow \infty$, то $W_{m}(p) \rightarrow 0$;

- если $p=p_{1}+a / \sqrt{m}$, то $W_{m}(p) \sim \Phi\left(-a / \varphi_{1, s}\left(p_{1}\right)-t_{\delta}\right)$;

- если $p=p_{2}+b / \sqrt{m}$, то $W_{m}(p) \sim \Phi\left(b / \varphi_{1, s}\left(p_{2}\right)-t_{\delta}\right)$;

- если $\left(p_{1}-p\right) \sqrt{m} \rightarrow \infty$ или $\left(p-p_{2}\right) \sqrt{m} \rightarrow \infty$, то $W_{m}(p) \rightarrow 1$.

Аналогичные результаты, базирующиеся на соотношениях (33), (34), имеют место и для $\mathrm{B \Pi} \Pi_{n}$.

\section{3. Точные доверительные интервалы}

Опишем алгоритм построения точного доверительного интервала для неизвестного размера $N$ однородной совокупности при ВП $\Pi_{n}\left(m_{1}, \ldots, m_{s}\right)$. Для этого прежде всего надо знать значения вероятностей (см. (2) или (6))

$$
a_{N}(l)=\mathbf{P}_{N}(\eta=l)=\Psi^{-1}(N)\left(\begin{array}{c}
N \\
l
\end{array}\right) \sum_{j=m}^{l}(-1)^{l-j}\left(\begin{array}{l}
l \\
j
\end{array}\right) \Psi_{s}(j), \quad m \leqslant l \leqslant n \wedge N,
$$

для достаточно большого набора значений параметра $N \geqslant m$, которые заполняют некоторую указанную на рисунке полосу.

Далее, при заданном доверительном уровне $\gamma=1-\delta$ для каждого $N$ определяем два целых числа $l_{1}(N)<l_{2}(N)$ так, чтобы выполнялось условие

$$
\mathbf{P}_{N}\left(l_{1}(N) \leqslant \eta \leqslant l_{2}(N)\right)=\sum_{l=l_{1}(N)}^{l_{2}(N)} a_{N}(l) \geqslant \gamma
$$


и при этом в сумму были включены все значения $l$ с наибольшими вероятностями их появления (при данном $N$ ). Так строится доверительная полоса $D_{\gamma}$ с границами $l_{i}(N), i=1,2$. Пусть теперь наблюдается значение $\eta$. Сечение на уровне $\eta$ полосы $D_{\gamma}$ определяет два числа $N_{1}(\eta)<N_{2}(\eta)$, а соответствующий интервал $\left[N_{1}(\eta), N_{2}(\eta)\right]$ и есть искомый $\gamma$-доверительный интервал, так как по построению

$$
\mathbf{P}_{N}\left(N_{1}(\eta) \leqslant N \leqslant N_{2}(\eta)\right)=\mathbf{P}_{N}\left(l_{1}(N) \leqslant \eta \leqslant l_{2}(N)\right) \geqslant \gamma
$$

Поскольку распределение $\eta$ асимметрично, то так построенный доверительный интервал будет короче обычного (центрального) интервала, когда границы $l_{i}(N)$ рассчитываются по правилу

$$
\mathbf{P}_{N}\left(\eta<l_{1}(N)\right) \leqslant \frac{\delta}{2}, \quad \mathbf{P}_{N}\left(\eta>l_{2}(N)\right) \leqslant \frac{\delta}{2} .
$$

Для вычисления вероятностей (40) можно использовать следующие рекуррентные соотношения [26]. Положим

$$
a\left(l ; m_{1}, \ldots, m_{k}\right)=\frac{1}{l !} \sum_{j=0}^{l-m_{(k)}}(-1)^{j}\left(\begin{array}{c}
l \\
j
\end{array}\right) \prod_{i=1}^{k}\left(\begin{array}{c}
l-j \\
m_{i}
\end{array}\right), \quad m_{(k)}=\max _{1 \leqslant i \leqslant k} m_{i}
$$

тогда формулу (40) можно записать в виде

$$
a_{N}(l)=\Psi_{s}^{-1}(N) \frac{N !}{(N-l) !} a\left(l ; m_{1}, \ldots, m_{s}\right) .
$$

Коэффициенты $a\left(l ; m_{1}, \ldots, m_{s}\right)$ удовлетворяют соотношениям

$$
\begin{aligned}
& a\left(l ; m_{1}, \ldots, m_{s}\right)=\sum_{k} \frac{1}{k !}\left(\begin{array}{c}
l-k \\
m_{s}-k
\end{array}\right) a\left(l-k ; m_{1}, \ldots, m_{s-1}\right), \\
& a\left(l ; m_{1}, \ldots, m_{s}\right)=\frac{l-m_{s}+1}{m_{s}} a\left(l ; m_{1}, \ldots, m_{s}-1\right)+\frac{1}{m_{s}} a\left(l-1 ; m_{1}, \ldots, m_{s}-1\right) .
\end{aligned}
$$

\section{4. Оценивание вероятности появления нового элемента}

Иногда основной интерес представляют те или иные конкретные параметрические функции от неизвестного состава исследуемой совокупности. Одной из таких интересных функций, которой уделялось значительное внимание в литературе, является вероятность появления нового элемента (в другой терминологии вероятность обнаружения нового вида) при последовательном выборе элементов с возвращением. Здесь мы рассмотрим простейший вариант задачи оценивания такой функции, когда элементы выбираются из совокупности $U$ по схеме равновероятной повторной выборки.

Обозначим вероятность появления нового элемента в $k$-м испытании через $\theta_{k}(N)(N-$ неизвестное общее число элементов $U)$, тогда, очевидно, для рассматриваемой схемы выбора

$$
\theta_{k}(N)=\left(1-\frac{1}{N}\right)^{k-1}, \quad k=1,2, \ldots
$$


Тем самым $\theta_{k}(N)$ представляет собой полином степени $k-1$ от $1 / N$. Мы предполагаем, что в нашем распоряжении имеется предварительная выборка объема $n$, и пусть, как обычно, $\mu_{r}=\mu_{r}(n)$ обозначает число элементов, появившихся в выборке ровно $r$ раз, $r=1, \ldots, n$. По этим исходным данным $\mu_{1}, \ldots, \mu_{n}$, как отмечено в п.3.3, при $k \leqslant n$ можно построить НОМД для $\theta_{k}(N)$, которая имеет вид

$$
\theta_{k}^{*}=\left(\Delta^{\eta} 0^{n}\right)^{-1} \sum_{j=0}^{k-1}(-1)^{j}\left(\begin{array}{c}
k-1 \\
j
\end{array}\right) \Delta^{\eta} 0^{n-j}
$$

где $\eta=\mu_{1}+\ldots+\mu_{n}$, либо линейную несмещенную оценку (которая является единственной в классе линейных оценок)

$$
\tilde{\theta}_{k}=\sum_{r=1}^{n} c_{r}(k, n) \mu_{r}
$$

где

$$
c_{r}(k, n)=\sum_{j=0}^{k-1}(-1)^{j}\left(\begin{array}{c}
k-1 \\
j
\end{array}\right) \frac{(r)_{j+1}}{(n)_{j+1}} .
$$

Нетрудно показать, что

$$
c_{r}(k, n)=\left(\begin{array}{l}
n-k \\
r-1
\end{array}\right)\left(\begin{array}{l}
n \\
r
\end{array}\right)^{-1}
$$

тем самым оценка (43) имеет вид

$$
\tilde{\theta}_{k}=\sum_{r=1}^{n-k+1}\left(\begin{array}{l}
n-k \\
r-1
\end{array}\right)\left(\begin{array}{l}
n \\
r
\end{array}\right)^{-1} \mu_{r}
$$

Если же $k>n$, то несмещенных оценок для $\theta_{k}(N)$ по выборке объема $n$ не существует.

Замечание 5. Если ввести числа Стирлинга 2 -го рода $S(n, r)=\Delta^{r} 0^{n} / r$, то формуле (42) можно придать вид

$$
\theta_{k}^{*}=\sum_{j=0}^{k-1}(-1)^{j}\left(\begin{array}{c}
k-1 \\
j
\end{array}\right) S(n-j, \eta) / S(n, \eta)
$$

а также

$$
\theta_{k}^{*}=\sum_{j=0}^{n-k}\left(\begin{array}{c}
n-k \\
j
\end{array}\right) S(k+j-1, \eta-1) / S(n, \eta)
$$

Представление (45) удобно использовать при малых значениях $k$, в то время как (46) более удобно для вычислений при значениях $k$, близких к $n$. В частности, из (46) следует, что $\theta_{n}^{*}=S(n-1, \eta-1) / S(n, \eta)$, соответствующая же линейная оценка (см. (44)) есть $\tilde{\theta}_{n}=\mu_{1} / n$. В обоих случаях для конкретных вычислений можно воспользоваться известными таблицами чисел Стирлинга (см., например, [20, c.62]). 
Замечание 6. Как показано в [39], результат, выраженный соотношением (44), остается в силе и для неравновероятной схемы выбора элементов при произвольных вероятностях $p_{1}, \ldots, p_{N}, p_{1}+\ldots+p_{N}=1$, извлечения соответствующих элементов совокупности в каждом испытании. Дальнейшие результаты для неравновероятной схемы см. в [36].

Как обычно, сравнение эффективностей оценок $\tilde{\theta}_{k}$ и $\theta_{k}^{*}$ удобно провести в асимптотической постановке. Именно, будем предполагать, что $N, n \rightarrow \infty$ так, что

$$
\alpha=\frac{n}{N} \in\left[\alpha_{0}, \alpha_{1}\right], \quad 0<\alpha_{0}<\alpha_{1}<\infty
$$

и при этом условии рассмотрим момент $k$, близкий к $n: k=n-s, s \leqslant C<\infty$ (такие значения $k$ представляют обычно наибольший практический интерес). В этих условиях оценивание $\theta_{k}(N)$ (см. (41)) фактически сводится к оцениванию функции $\psi(\alpha)=e^{-\alpha}$, так как

$$
\theta_{n-s}(N)=\psi(\alpha)(1+O(1 / n))
$$

Рассмотрим сначала линейную оценку $\tilde{\theta}_{k}$. Из (41) и из известных результатов в классической задаче о размещении [19, с.56] следует, что в условиях (47) с вероятностью, стремящейся к 1 при $n \rightarrow \infty$,

$$
\tilde{\theta}_{n-s}=\frac{\mu_{1}}{n}\left(1+O\left(\frac{1}{n}\right)\right)
$$

и при этом

$$
\mathcal{L}\left(\tilde{\theta}_{n-s}\right) \sim \mathcal{N}\left(\psi(\alpha), \sigma_{1}^{2}(\alpha) / n\right)
$$

где $\sigma_{1}^{2}(\alpha)=e^{-\alpha}\left(1-e^{-\alpha}\left(1-\alpha+\alpha^{2}\right)\right)$.

Что же касается оценки $\theta_{k}^{*}$, то из (46) для нее можно вывести следующее асимптотическое представление, также справедливое с вероятностью, близкой к 1, в рассматриваемых условиях: $\theta_{n-s}^{*}=\psi\left(\hat{\alpha}_{n}\right)(1+O(1 / n))$, где $\hat{\alpha}_{n}$ определено в (33). Отсюда и из (34) следует, что

$$
\mathcal{L}\left(\theta_{n-s}^{*}\right) \sim \mathcal{N}\left(\psi(\alpha), \sigma_{0}^{2}(\alpha) / n\right),
$$

где $\sigma_{0}^{2}(\alpha)=\alpha^{3} e^{-3 \alpha} /\left(1-e^{-\alpha}(1+\alpha)\right)$.

Подчеркнем, что фактически НОМД $\theta_{n-s}^{*}$ совпадает с асимптотической ОМП $\hat{\theta}_{n-s}=\psi(\hat{\alpha})$.

Еще один способ асимптотического оценивания $\theta_{k}(N)$, учитывающий представление (48), состоит в замене $\alpha$ линейной оценкой (см. п.3.3)

$$
\tilde{\alpha}_{n}=\frac{1}{n-1} \sum_{r=2}^{n} r(r-1) \mu_{r} .
$$

Эта оценка исследована в [12], где установлено, что в условиях (47) она асимптотически нормальна $\mathcal{N}(\alpha, 2 \alpha / n)$. Отсюда следует, что оценка $\theta_{n-s}^{\prime}=\psi\left(\tilde{\alpha}_{n}\right)$ асимптотически нормальна с параметрами $\psi(\alpha)$ и $\sigma_{2}^{2}(\alpha) / n$, где $\sigma_{2}^{2}(\alpha)=2 \alpha e^{-2 \alpha}$. 
Таблица 1. Значения $e_{i}(\alpha), i=1,2$

\begin{tabular}{c|llllllllll}
\hline$\alpha$ & 0.1 & 0.2 & 0.3 & 0.4 & 0.5 & 0.6 & 0.7 & 0.8 & 0.9 & 1.0 \\
\hline$e_{1}(\alpha)$ & 0.991 & 0.988 & 0.967 & 0.952 & 0.935 & 0.926 & 0.893 & 0.868 & 0.841 & 0.810 \\
$e_{2}(\alpha)$ & 0.967 & 0.934 & 0.903 & 0.871 & 0.841 & 0.810 & 0.781 & 0.752 & 0.724 & 0.696 \\
\hline$\alpha$ & 1.2 & 1.4 & 1.6 & 1.8 & 2.0 & 2.2 & 2.4 & 2.6 & 2.8 & 3.0 \\
\hline$e_{1}(\alpha)$ & 0.742 & 0.664 & 0.582 & 0.497 & 0.415 & 0.340 & 0.272 & 0.215 & 0.167 & 0.128 \\
$e_{2}(\alpha)$ & 0.643 & 0.592 & 0.544 & 0.498 & 0.458 & 0.416 & 0.378 & 0.343 & 0.310 & 0.278
\end{tabular}

Таблица 2. Значения $a^{-1}(t)$

\begin{tabular}{|c|c|c|c|c|c|}
\hline$t$ & $a^{-1}(t)$ & $t$ & $a^{-1}(t)$ & $t$ & $a^{-1}(t)$ \\
\hline 0.05 & 20.00000 & $\overline{0.40}$ & 2.23161 & $\overline{0.72}$ & 0.69732 \\
\hline 0.10 & 9.99955 & 0.42 & 2.08498 & 0.74 & 0.63578 \\
\hline 0.12 & 8.33133 & 0.44 & 1.94908 & 0.76 & 0.57649 \\
\hline 0.14 & 7.13718 & 0.46 & 1.82260 & 0.78 & 0.51935 \\
\hline 0.16 & 6.23779 & 0.48 & 1.70442 & 0.80 & 0.46421 \\
\hline 0.18 & 5.53360 & 0.50 & 1.59362 & 0.82 & 0.41096 \\
\hline 0.20 & 4.96511 & 0.52 & 1.48941 & 0.84 & 0.35946 \\
\hline 0.22 & 4.49469 & 0.54 & 1.39112 & 0.86 & 0.30963 \\
\hline 0.24 & 4.09744 & 0.56 & 1.29815 & 0.88 & 0.26136 \\
\hline 0.26 & 3.75627 & 0.58 & 1.21001 & 0.90 & 0.21456 \\
\hline 0.28 & 3.45908 & 0.60 & 1.12626 & 0.92 & 0.16915 \\
\hline 0.30 & 3.19706 & 0.62 & 1.04652 & 0.94 & 0.12505 \\
\hline 0.32 & 2.96366 & 0.64 & 0.97045 & 0.96 & 0.08221 \\
\hline 0.34 & 2.75388 & 0.66 & 0.89775 & 0.98 & 0.04054 \\
\hline 0.36 & 2.56387 & 0.68 & 0.82816 & 1.00 & 0.00000 \\
\hline 0.38 & 2.39059 & 0.70 & 0.76143 & & \\
\hline
\end{tabular}

Таким образом, все три способа асимптотического оценивания величины $\theta_{n-s}(N)$ приводят к с.а.н. оценкам, различающимся лишь величинами асимптотических дисперсий; при этом $\sigma_{0}^{2}(\alpha)<\sigma_{i}^{2}(\alpha), i=1,2$. Сравнение же $\sigma_{1}^{2}(\alpha)$ и $\sigma_{2}^{2}(\alpha)$ показывает, что $\sigma_{1}^{2}(\alpha)<\sigma_{2}^{2}(\alpha)$ при $\alpha<\alpha^{*}=1,793 \ldots$ и $\sigma_{1}^{2}(\alpha)>\sigma_{2}^{2}(\alpha)$ при $\alpha>\alpha^{*}$. Следовательно, при малых значениях $\alpha$ оценка $\tilde{\theta}_{n-s}$ более эффективна, нежели $\theta_{n-s}^{\prime}$, а при $\alpha>\alpha^{*}$ более точной из этих двух оценок является оценка $\theta_{n-s}^{\prime}$. Наконец, эффективность каждой из этих двух простых оценок по отношению к оптимальной оценке $\theta_{n-s}^{*}$ можно измерять соответствующими отношениями $e_{i}(\alpha)=\sigma_{0}^{2}(\alpha) / \sigma_{i}^{2}(\alpha), i=1,2$. Ниже приводится таблица значений этих эффективностей и таблица значений функции $a^{-1}(t)$. 


\section{5. Цензурированные данные}

При ОСВ мы получаем данные в частотной форме, и до сих пор обсуждалась ситуация, так сказать, с полными данными, когда все положительные частоты исходов наблюдаемы. Альтернативной является ситуация с неполными (или цензурированными) данными, т.е. когда наблюдению доступны не все положительные частоты, а лишь те из них, значения которых, например, принадлежат некоторому подмножеству $R$ натуральных чисел. Цензурирование наблюдений вносит, как известно, новые существенные аспекты в исследуемую проблематику, связанные как с особенностями самого эффекта цензурирования, так и с необходимостью модернизации соответствующих методик исследования. Применительно к рассматриваемому случаю цензурирование практически происходит, например, когда регистрирующее результаты испытаний устройство имеет некоторый порог чувствительности, так что если накопленные частоты каких-то исходов не достигают этого порога, то они не регистрируются, т.е. для статистика они становятся ненаблюдаемыми. Для этой ситуации $R=R_{m}=\{m+1, m+2, \ldots\}$ при некотором целом $m>0$, и это, возможно, наиболее практически интересный случай, который ниже анализируется более детально.

В работе [17] обсуждалась проблема оценивания по цензурированным данным неизвестного размера $N$ конечной совокупности в схеме простой повторной выборки $\Pi_{n}$. В этом случае для любого подмножества $R \subset \mathbf{N}=\{1,2, \ldots\}$ достаточной статистикой для параметра $N$ является пара $(\eta(R), \zeta(R))$, где

$$
\eta(R)=\sum_{r \in R} \mu_{r}, \quad \zeta(R)=\sum_{r \in R} r \mu_{r}
$$

(для полных данных, т.е. когда $R=\mathbf{N}$, величина $\zeta=n$ ).

Для больших выборок, т.е. когда $n, N \rightarrow \infty$ и $0<\alpha_{0} \leqslant \alpha=n / N \leqslant \alpha_{1}<$ $\infty$, для распределения достаточной статистики справедливо равномерное по $\alpha$ асимптотическое соотношение

$$
\mathcal{L}(\eta(R) / n, \zeta(R) / n) \sim \mathcal{N}\left(\left(m_{1}(\alpha) / \alpha, m_{2}(\alpha) / \alpha\right),\left\|\sigma_{i j}(\alpha)\right\|_{1}^{2} /(n \alpha)\right),
$$

где

$$
\begin{aligned}
m_{j}(\alpha) & =\sum_{r \in R} r^{j-1} \pi_{r}(\alpha), \quad j=1,2,3, \\
\pi_{r}(\alpha) & =\exp (-\alpha) \alpha^{r} / r !, \quad r=0,1, \ldots, \\
\sigma_{11}(\alpha) & =m_{1}(\alpha)\left(1-m_{1}(\alpha)\right)-\frac{1}{\alpha}\left(m_{2}(\alpha)-\alpha m_{1}(\alpha)\right)^{2}, \\
\sigma_{12}(\alpha) & =m_{2}(\alpha)\left(1-m_{1}(\alpha)\right)-\frac{1}{\alpha}\left(m_{2}(\alpha)-\alpha m_{1}(\alpha)\right)\left(m_{3}(\alpha)-\alpha m_{2}(\alpha)\right), \\
\sigma_{22}(\alpha) & =m_{3}(\alpha)-m_{2}^{2}(\alpha)-\frac{1}{\alpha}\left(m_{3}(\alpha)-\alpha m_{2}(\alpha)\right)^{2} .
\end{aligned}
$$

На соотношении (52) основан следующий общий алгоритм состоятельного оценивания неизвестного параметра $\alpha$. Пусть $g(x, y)$ - произвольная гладкая функция двух переменных такая, что функция $H(\alpha)=g\left(m_{1}(\alpha) / \alpha, m_{2}(\alpha) / \alpha\right)$ является монотонной $\left(H^{\prime}(\alpha)>0\right)$. Обозначим класс всех таких функций $H(\alpha)$ через 
$\mathcal{H}$. Тогда для произвольной функции $H \in \mathcal{H}$ в рассматриваемых асимптотических условиях

$$
\mathcal{L}\left(H_{n}\right) \sim \mathcal{N}\left(H(\alpha), \sigma_{g}^{2}(\alpha) /(n \alpha)\right)
$$

где

$$
\begin{aligned}
H_{n} & =g(\eta(R) / n, \zeta(R) / n), \\
\sigma_{g}^{2}(\alpha) & =g_{x}^{2}(\alpha) \sigma_{11}(\alpha)+2 g_{x}(\alpha) g_{y}(\alpha) \sigma_{12}(\alpha)+g_{y}^{2}(\alpha) \sigma_{22}(\alpha)
\end{aligned}
$$

и $g_{x}(\alpha), g_{y}(\alpha)$ - соответствующие производные функции $g(x, y)$, вычисленные при $x=m_{1}(\alpha) / \alpha, y=m_{2}(\alpha) / \alpha$.

Поскольку определена обратная функция $H^{-1}(t)$, отсюда следует, что

$$
\mathcal{L}\left(\alpha_{n}\right) \sim \mathcal{N}\left(\alpha, v_{g}^{2}(\alpha) / n\right),
$$

где $\alpha_{n}=H^{-1}\left(H_{n}\right), v_{g}^{2}(\alpha)=\sigma_{g}^{2}(\alpha) /\left(\alpha H^{\prime}(\alpha)\right)^{2}$.

Соотношение (53) и является итоговым. Во-первых, оно означает, что статистика $\alpha_{n}$ является с.а.н. оценкой $\alpha$ с асимптотической дисперсией порядка $n^{-1}$. Во-вторых, оно дает возможность рассчитать и асимптотический доверительный интервал для $\alpha$ : при доверительном уровне $1-\delta$ такой интервал имеет вид $\left(\alpha_{n} \pm t_{\delta / 2} v_{g}\left(\alpha_{n}\right) / \sqrt{n}\right)$.

Отметим, что в описанной методике оптимальное решение для общего случая задания множества $R$ получить трудно. Проблема состоит в отыскании для нормального семейства со связанными параметрами функции $H^{*} \in \mathcal{H}$, минимизирующей функционал $v_{g}^{2}(\alpha)$ в $(53)$.

Для отмеченного выше случая $R=R_{m}$ изложенный алгоритм конкретизируется следующим образом. Обозначим

$$
\Pi_{m}(\alpha)=1-\bar{\Pi}_{m}(\alpha)=\sum_{r=0}^{m} \pi_{r}(\alpha),
$$

тогда в выражении (52) $m_{1}(\alpha)=\bar{\Pi}_{m}(\alpha), m_{2}(\alpha)=\alpha \bar{\Pi}_{m-1}(\alpha)$. Возьмем $g(x, y)=$ $g_{a}(x, y)=y-a x, a \geqslant 0$. Тогда $H(\alpha)=H_{a}(\alpha)=\left(m_{2}(\alpha)-a m_{1}(\alpha)\right) / \alpha$ и

$$
H_{a}^{\prime}(\alpha)=\left(m(m+1-a) \pi_{m+1}(\alpha)+a \bar{\Pi}_{m+1}(\alpha)\right) / \alpha^{2} .
$$

Отсюда следует, что при любом $a \in[0, m+1]$ функция $H_{a} \in \mathcal{H}$. В частности, при $a=0$ имеем простую оценку $\alpha_{n}^{(0)}=\bar{\Pi}_{m-1}^{-1}(\zeta(R) / n)$ с асимптотической дисперсией $v_{g_{0}}^{2}(\alpha) / n$, где $($ см. $(52)) v_{g_{0}}^{2}(\alpha)=\sigma_{22}(\alpha) /\left(\alpha\left(\pi_{m-1}(\alpha)\right)^{2}\right)$ и

$$
\sigma_{22}(\alpha) / \alpha=\alpha \bar{\Pi}_{m-2}(\alpha)+\bar{\Pi}_{m-1}(\alpha)-\alpha \bar{\Pi}_{m-1}^{2}(\alpha)-\left(\alpha \pi_{m-1}(\alpha)+\bar{\Pi}_{m-1}(\alpha)\right)^{2} .
$$

Другим естественным вариантом является выбор функции $g(x, y)$ вида $f(y / x)$. Тогда из (52) следует, что отношение $\zeta(R) / \eta(R)$ будет иметь асимптотически нормальное распределение со средним

$$
m(\alpha)=m_{2}(\alpha) / m_{1}(\alpha)=\alpha \bar{\Pi}_{m-1}(\alpha) / \bar{\Pi}_{m}(\alpha),
$$

и так как функция $m(\alpha)$ монотонна при $|R|>1$, статистика $\alpha_{n}^{(1)}=m^{-1}(\zeta(R) / \eta(R))$ также будет с.а.н. оценкой $\alpha$. Вопрос о сравнении оценок $\alpha_{n}^{(0)}$ и $\alpha_{n}^{(1)}$ остается пока открытым. 


\section{6. Моменты остановки}

Большой круг задач, связанных с оцениваем состава конечной совокупности, порождают выборочные планы со случайным числом наблюдений (испытаний), определяемым тем или иным правилом остановки. В качестве примера такого ВП рассмотрим следующую модель эксперимента, которая получила в литературе название распространение слухов (см., например, [21, с.69]). Именно, пусть имеется коллектив из $N$ лиц, среди которых распространяется некоторая новость. В начальный момент новость сообщается некоторому числу лиц $Y_{1}$. В следующий момент каждое из этих $Y_{1}$ лиц независимо от остальных передает ее какому-нибудь другому лицу, выбираемому с равной вероятностью из всех остальных $N-1$ членов коллектива. В результате таких $Y_{1}$ контактов новость становится известной некоторому дополнительному числу лиц $Y_{2} \quad\left(\leqslant Y_{1}\right)$, которые по описанному алгоритму продолжают распространение слуха на третьем этапе и т.д. Таким образом, процесс распространения слуха может быть описан невозрастающей последовательностью дискретных случайных величин $\left\{Y_{1} \geqslant Y_{2} \geqslant \ldots\right\}$, где $Y_{t}$ есть число лиц, узнающих новость в результате случайных контактов на $t$-ом этапе, $t=2,3, \ldots$ Поскольку $Y_{1}+Y_{2}+\ldots \leqslant N$, а размер $N$ коллектива конечен, то последовательность $Y_{t}$ также конечна. Пусть $\nu=\min \left\{t: Y_{t}=0\right\}$ есть момент прекращения распространения слуха. Предполагается, что последовательность $\left\{Y_{1}, \ldots, Y_{\nu}\right\}$ полностью наблюдаема, и по этой информации требуется оценить неизвестный размер $N$ коллектива. Эта задача рассматривалась в [8] и [27], где получены следующие результаты.

Полной достаточной статистикой для параметра $N$ является в данном случае $S_{\nu}=Y_{1}+\ldots+Y_{\nu}$ - общее число лиц, которым в конечном итоге станет известна новость. Закон распределения статистики $S_{\nu}$ имеет вид (при $Y_{1}=r$ )

$$
\mathbf{P}_{N}\left(S_{\nu}=s\right)=p_{N}(s \mid r)=\frac{(N-r) !(s-1)}{(N-s) !(N-1)^{s}} S(s-1, s-r ; r-1), \quad s=r, \ldots, N
$$

где

$$
S(n, k ; x)=\frac{1}{k !} \sum_{j=1}^{k}(-1)^{k-j}\left(\begin{array}{l}
k \\
j
\end{array}\right)(x+j)^{n}=\frac{\Delta^{k} x^{n}}{k !}
$$

есть введенные и исследованные в [10] обобщенные числа Стирлинга 2-го рода. Отсюда находится следующая НОМД $N_{r}^{*}$ для $N$ :

$$
N_{r}^{*}=r+\varphi_{r}\left(S_{\nu}\right)
$$

где функция $\varphi_{r}(s)=S(s-1, s-r-1 ; r) / S(s-1, s-r ; r-1)$. В частности,

$$
N_{1}^{*}=\frac{1}{2} S_{\nu}\left(S_{\nu}-1\right)+1, \quad N_{2}^{*}=\frac{1}{12}\left(S_{\nu}-2\right)\left(3 S_{\nu}+7\right)+2 .
$$

Если наблюдаемое значение $S_{\nu}$ велико, то вместо (54) можно использовать простую приближенную формулу $N_{r}^{*} \approx S_{\nu}^{2} /(2 r)$.

Если значение $N$ велико, то при любом фиксированном начальном значении $Y_{1}=r$ случайная величина $S_{\nu} / \sqrt{N}$ имеет предельное распределение с плотностью

$$
f(x \mid r)=\frac{x^{2 r-1}}{(r-1) ! 2^{r-1}} \exp \left(-x^{2} / 2\right), \quad x>0
$$


при этом имеет место и соответствующая сходимость ее моментов любого порядка. В частности,

$$
\mathbf{E}\left(S_{\nu} / \sqrt{N}\right) \rightarrow a_{r}=\int_{0}^{\infty} x f(x \mid r) d x=\sqrt{\pi / 2} \frac{\Gamma(2 r)}{4^{r-1} \Gamma^{2}(r)}
$$

и

$$
\frac{a_{r}}{a_{r-1}}=1+\frac{1}{2(r-1)}
$$

Отсюда видно, что увеличение начального значения приводит к увеличению в среднем и $S_{\nu}$, однако с увеличением $Y_{1}$ этот рост становится все менее значительным. Так, переход от $Y_{1}=1 \mathrm{k} Y_{1}=2$ увеличивает $S_{\nu}$ в среднем на $50 \%$, а переход от $Y_{1}=5$ к $Y_{1}=6$ приводит к увеличению $S_{\nu}$ в среднем лишь на $10 \%$.

Наконец, при $N \rightarrow \infty$ и фиксированном $r$ статистика $2 r N_{r}^{*} / N$ распределена асимптотически по закону $\chi^{2}$ с $2 r$ степенями свободы; при этом $\mathbf{D} N_{r}^{*} \sim N^{2} / r$, а асимптотическим $\gamma$-доверительным интервалом для $N$ является интервал $\left(S_{\nu}^{2} / \chi_{(1+\gamma) / 2,2 r}^{2}, S_{\nu}^{2} / \chi_{(1-\gamma) / 2,2 r}^{2}\right)$. Таким образом, оценка $N_{r}^{*}$ имеет большой разброс: длина доверительного интервала имеет такой же порядок, как и сама оценка (см. (55)).

Шелый класс выборочных планов со случайным числом испытаний образуют эксперименты, в которых момент остановки связан с временем ожидания до достижения заданной конфигурации заполнения ячеек (см. п.2). Общее определение такого ВП следующее: пусть комплекты частиц размещаются в $N=N_{1}+\ldots+N_{k}$ ячейках последовательно до тех пор, пока впервые в $j$-й группе появятся не менее $l_{j}$ ячеек, заполненных не менее $r_{j}$ частицами каждая, $j=1, \ldots, k$. Здесь ВП определяется (помимо размеров комплектов $m_{1}, m_{2}, \ldots$ ) набором пар целых чисел $\left.\left(l_{j}, r_{j}\right), j=1, \ldots, k\right)$, а наблюдаемые данные есть число размещенных комплектов (время ожидания) и заполнения непустых ячеек (в случае модели с полными данными) или числа ячеек с заданным заполнением (в случае цензурированных данных). В такой общей постановке задача оценивания неизвестного состава $\bar{N}=\left(N_{1}, \ldots, N_{k}\right)$ на сегодня остается открытой. Что касается времени ожидания в полиномиальной схеме размещения $\left(\right.$ все $\left.m_{i}=1\right)$, то обзор последних результатов имеется в [7], см. также [9].

\section{7. Минимаксное оценивание}

Что касается минимаксного подхода в рассматриваемой проблематике, то отметим интересную работу [41], в которой рассматривается близкая к обсуждаемым здесь задача оценивания вектора долей $\bar{\beta}=\left(\beta_{j}=N_{j} / N, j=1, \ldots, k\right)$ $k$-слойной совокупности (общий размер $N$ также неизвестен) по одной бесповторной выборке $\bar{\Pi}_{n}$. В этой работе для $\bar{\beta}$ построена минимаксная оценка при общей функции потерь вида

$$
L(\bar{\beta}, \bar{d})=\sum_{i, j=1}^{k} c_{i j}\left(\beta_{i}-d_{i}\right)\left(\beta_{j}-d_{j}\right)
$$

где $\bar{d}=\left(d_{1}, \ldots, d_{k}\right)$ - оценка $\bar{\beta}$ и $\left\|c_{i j}\right\|_{1}^{k}$ - произвольная неотрицательно определенная матрица. При такой функции потерь оценка имеет вид

$$
d_{i}(\bar{\eta})=\left(\eta_{i}+p_{i}^{0} \sqrt{n}\right) /(n+\sqrt{n}), \quad i=1, \ldots, k,
$$


где

$$
\begin{aligned}
\vec{p}^{0} & =\left(p_{1}^{0}, \ldots, p_{k}^{0}\right)=\arg \max _{\bar{p} \in \mathcal{P}}\left\{\sum_{i=1}^{k} c_{i i} p_{i}-\sum_{i, j=1}^{k} c_{i j} p_{i} p_{j}\right\} \\
\mathcal{P} & =\left\{\bar{p}=\left(p_{1}, \ldots, p_{k}\right): p_{i} \geqslant 0, i=1, \ldots, k, p_{1}+\ldots+p_{k}=1\right\} .
\end{aligned}
$$

В частности, при $k=2$ отсюда следует минимаксная оценка $d(X)=(X+$ $\sqrt{n} / 2) /(n+\sqrt{n})$ параметра $M / N$ гипергеометрического распределения

$$
\mathbf{P}(X=x)=\left(\begin{array}{c}
M \\
x
\end{array}\right)\left(\begin{array}{c}
N-M \\
n-x
\end{array}\right) /\left(\begin{array}{l}
N \\
n
\end{array}\right)
$$

при неизвестных $M$ и $N(\geqslant n)$ и квадратичной функции потерь $L(M / N, d)=$ $(M / N-d)^{2}$.

С помощью аналогичного подхода в [40] построена минимаксная оценка вектора состава $\bar{N}=\left(N_{1}, \ldots, N_{k}\right)$ при известном общем размере $N$ совокупности (ВП есть по-прежнему $\bar{\Pi}_{n}$ ). В частности, при $k=2$ получаем хорошо известный результат [31], состоящий в том, что

$$
d(X)=N\left(X+\frac{1}{2} \sqrt{n \frac{N-n}{N-1}}\right) /\left(n+\sqrt{n \frac{N-n}{N-1}}\right)
$$

есть минимаксная оценка (неизвестного) параметра $M$ гипергеометрического распределения (56) при квадратичной функции потерь.

В $[40,41,42]$ имеются и другие результаты по минимаксному оцениванию, а также ссылки ніа соответствующую литературу.

\section{8. Байесовское оценивание}

Примером байесовского подхода к оцениванию неизвестного размера $N$ конечной совокупности является статья [35] (см. также [38]), в которой рассматривается полиномиальная схема испытаний с некоторыми (неизвестными) вероятностями $p_{1}, \ldots, p_{N}, p_{1}+\ldots+p_{N}=1$, наблюдения соответствующих элементов. При этом априорное совместное распределение параметров $N, p_{1}, \ldots, p_{N}$ задается плотностью $\mathbf{P}(N) f\left(p_{1}, \ldots, p_{N} \mid N\right)$, где

$$
f\left(p_{1}, \ldots, p_{N} \mid N\right)=\frac{\Gamma(k N)}{\Gamma^{N}(k)} \prod_{i=1}^{N} p_{i}^{k-1}
$$

есть $N$-мерная плотность Дирихле, а

$$
\mathbf{P}(N)=\left(\begin{array}{c}
N+r-1 \\
N
\end{array}\right) \alpha^{r}(1-\alpha)^{N} /\left(1-\alpha^{N}\right), \quad 0<\alpha \leqslant 1, \quad N \geqslant 1
$$

есть усеченное в нуле отрицательное биномиальное распределение (параметры $k, \alpha$ и $r$ считаются неизвестными).

В [35] построена байесовская интервальная оценка для $N$, даются некоторые рекомендации по выбору параметров $k, \alpha$ и $r$ и эти результаты применяются к оценке числа видов насекомых, обитающих в горной Кении. 


\section{9. Повторный выбор из совокупности с занумерованными элементами}

В ряде ситуаций элементы исследуемой совокупности могут быть занумерованы числами от 1 до $N$, причем общее их число $N$ по-прежнему считается неизвестным. В этом случае номер наблюдаемого элемента несет дополнительную информацию об общем числе элементов: если наблюдавшийся элемент имеет номер $N_{1}$, то отсюда для $N$ следует оценка $N \geqslant N_{1}$. Таким образом, это существенно другая (в сравнении с рассматривавшейся до этого) ситуация, и мы кратко обсудим здесь возможные подходы к оцениванию параметра $N$ для некоторых выборочных планов.

Рассмотрим сначала ВП простой повторной выборки $\Pi_{n}$. Игровую интерпретацию этой ситуации дает В. Феллер [21, с.231], предлагая для оценки числа зарегистрированных в городе автомобилей встать на перекрестке и записывать номера проезжающих автомобилей $X_{1}, \ldots, X_{n}$. В этом случае максимальный наблюденный номер $X_{(n)}=\max \left(X_{1}, \ldots, X_{n}\right)$ является полной достаточной статистикой для параметра $N$ с распределением

$$
g(t ; N)=\mathbf{P}_{N}\left(X_{(n)}=t\right)=\left(\frac{t}{N}\right)^{n}-\left(\frac{t-1}{N}\right)^{n}, \quad t=1,2, \ldots, N,
$$

(см., например, $[18$, с.163-164]) и для оценивания параметрических функций $\tau(N)$ можно использовать принцип достаточности, в соответствии с которым отыскание НОМД для $\tau(N)$ сводится к решению уравнения несмещенности

$$
\mathbf{E}_{N} u\left(X_{(n)}\right)=\tau(N) \quad \forall N \geqslant 1
$$

Эффективным приемом решения уравнения (58) является метод подгонки, суть которого состоит в следующем. Предположим, что отношение $g(t ; N) / \tau(N)$ можно представить в виде $v(t) g_{1}(t ; N)$, где $g_{1}(t ; N)$ принадлежит тому же семейству распределений, что и $g(t ; N)$. Тогда из полноты этого семейства следует, что уравнению (58) удовлетворяет единственная функция $u(t)=v^{-1}(t)$, что следует из цепочки тождеств (по $N$ )

$$
1 \equiv \sum_{t} u(t) g(t ; N) / \tau(N) \equiv \sum_{t} u(t) v(t) g_{1}(t ; N) \equiv \sum_{t} g_{1}(t ; N)
$$

Таким образом, в этом случае НОМД для $\tau(N)$ существует и имеет вид

$$
\tau^{*}=v^{-1}\left(X_{(n)}\right)
$$

Замечание 7. Метод подгонки, очевидно, можно применять в любых моделях, в том числе и непрерывных.

Применим метод подгонки для оценивания в нашем случае функции $\tau_{r}(N)=$ $N^{r}$ при целом $r>-n$. Тогда уравнение (58) с учетом (57) можно записать в виде

$$
\sum_{t} u(t) \frac{t^{n}-(t-1)^{n}}{t^{n+r}-(t-1)^{n+r}}\left(\left(\frac{t}{N}\right)^{n+r}-\left(\frac{t-1}{N}\right)^{n+r}\right)=1
$$


Отсюда сразу находим, что НОМД (59) есть в данном случае

$$
\tau_{r}^{*}=\frac{\left(X_{(n)}\right)^{n+r}-\left(X_{(n)}-1\right)^{n+r}}{\left(X_{(n)}\right)^{n}-\left(X_{(n)}-1\right)^{n}}=\left(X_{(n)}\right)^{r} \frac{1-\left(1-\left(X_{(n)}\right)^{-1}\right)^{n+r}}{1-\left(1-\left(X_{(n)}\right)^{-1}\right)^{n}} .
$$

При больших значениях $X_{(n)}$ вместо (60) можно использовать простое приближение $\left.\tau_{r}^{*} \approx(n+r) X_{(n)}\right)^{r} / n$. В частности, НОМД для размера совокупности $N$ имеет вид

$$
N^{*}=\tau_{1}^{*}=X_{(n)} \frac{1-\left(1-\left(X_{(n)}\right)^{-1}\right)^{n+1}}{1-\left(1-\left(X_{(n)}\right)^{-1}\right)^{n}} \approx \frac{n+1}{n} X_{(n)},
$$

а НОМД для дисперсии $\mathbf{D} N^{*}$ есть

$$
\begin{aligned}
\mathbf{D}^{*} N^{*} & =\left(N^{*}\right)^{2}-\left(N^{2}\right)^{*}=\left(\tau_{1}^{*}\right)^{2}-\tau_{2}^{*} \\
& =\left(X_{(n))}^{2}\left(\left(\frac{1-\left(1-\left(X_{(n)}\right)^{-1}\right)^{n+1}}{1-\left(1-\left(X_{(n)}\right)^{-1}\right)^{n}}\right)^{2}-\frac{1-\left(1-\left(X_{(n)}\right)^{-1}\right)^{n+2}}{1-\left(1-\left(X_{(n)}\right)^{-1}\right)^{n}}\right) \approx \frac{1}{n^{2}}\left(X_{(n)}\right)^{2} .\right.
\end{aligned}
$$

Иллюстрацией этой статистической процедуры являются следующие результаты, полученные моделированием с помощью таблицы 7.1а [2] случайных чисел $\left[18\right.$, с.23-24]: при $N=10000, n=10$ значения оценки $N^{*} \approx X_{(n)}(n+1) / n$ для десяти независимых выборок $X_{1}, \ldots, X_{n}$ оказались равными с точностью до единицы $10549,9841,10486,10891,10462,10873,8789,10753,9960,10287$, а значения $\sqrt{\mathbf{D}^{*} N^{*}} \approx X_{(n)} / n$ соответственно равны $959,895,953,990,951,988,799,978,906$, 935. Эти данные свидетельствуют о хорошем качестве оценок (61).

\subsection{0. Бесповторный выбор из совокупности с занумерован- ными элементами}

На том же пути в случае ВП простой бесповторной выборки $\bar{\Pi}_{n}$ можно показать, что снова полной достаточной статистикой является $X_{(n)}$ и

$$
g(t ; N)=\mathbf{P}_{N}\left(X_{(n)}=t\right)=\left(\begin{array}{c}
t-1 \\
n-1
\end{array}\right) /\left(\begin{array}{l}
N \\
n
\end{array}\right), \quad t=n, n+1, \ldots, N .
$$

Метод подгонки для оценивания функции $\tau_{r}(N)=(N+r) ! / N !, r>-n-$ целое число, приводит в данном случае к уравнению

$$
\frac{n}{n+r} \sum_{t} u(t) \frac{(t-1) !}{(t+r-1) !}\left(\begin{array}{c}
t+r-1 \\
n+r-1
\end{array}\right) /\left(\begin{array}{c}
N+r \\
n+r
\end{array}\right)=1 .
$$

Отсюда следует, что НОМД (59) в данном случае имеет вид

$$
\tau_{r}^{*}=\frac{n+r}{n} \frac{\left(X_{(n)}+r-1\right) !}{\left(X_{(n)}-1\right) !}
$$

В частности, НОМД для $N$ есть

$$
N^{*}=\frac{n+1}{n} X_{(n)}-1
$$

а НОМД для дисперсии $\mathbf{D} n^{*}=(N+1)(N-n) /(n(n+2))$ имеет вид

$$
\mathbf{D}^{*} N^{*}=\left(N^{*}\right)^{2}-\left(N^{2}\right)^{*}=X_{(n)}\left(X_{(n)}-n\right) / n^{2} \text {. }
$$




\section{Список литературы}

1. Беляев Ю. К. Вероятностные методы въборочного контроля. Наука, Москва, 1975.

2. Большев Л. Н., Смирнов Н. В. Таблицы математической статистики. Наука, Москва, 1983.

3. Воинов В. Г., Никулин М. С. Несмещенные оценки и их применения. Наука, Москва, 1989.

4. Иванов В. А., Ивченко Г. И., Медведев Ю. И. Дискретные задачи в теории вероятностей. В кн.: Итоги науки и техники, Сер. Теория вероятн., мат. статист., теор. киберн. 22. ВИНИТИ, Москва, 1984, с. 3-60.

5. Ивченко Г. И. О некоторых задачах статистического вывода для расслоенных совокупностей. Теория вероятностей и ее применения. (1984) 29, №1, 113-118.

6. Ивченко Г. И. Об оценивании параметров расслоенной конечной совокупности. В кн.: Статистические методы оценивания и проверки әипотез. Перм. ун-т, Пермь, 1986, c.11-14.

7. Ивченко Г. И. Время ожидания и связанные с ним характеристики в полиномиальной схеме. Дискретная математика (1993) 5, №3, 3-34.

8. Ивченко Г. И., Воскресенский В. Н. Об одной модели распространения слухов и ее применение в статистике. В кн.: Материаль XVI Всес. иколь-коллоквиума по теории вероятн. и матем. статист., Бакуриани. Тбилиси, 1982, с. 21-31.

9. Ивченко Г. И., Иванова Т. В. О случайном заполнении таблиц. Труды МИЭМ (1975) 44, 111-130.

10. Ивченко Г. И., Медведев Ю. И. Асимптотические представления конечных разностей от степенной функции в произвольной точке. Теория вероятностей и ее применения (1965) 10, №1, 151-156.

11. Ивченко Г. И., Медведев Ю. И. Некоторые многомерные теоремы в классической задаче о размещении. Теория вероятностей и ее применения (1965) 10, №1, 156-162.

12. Ивченко Г. И., Тимонина Е. Е. Об оценивании при выборе из конечной совокупности. Матем. заметки (1980) 28, №4, 623-633.

13. Ивченко Г. И., Тимонина Е. Е. О некоторых задачах оценивания для выборок из конечной совокупности. Изв. АН УзССР, Сер. физ.-матем. наук (1981) №4, 27-33.

14. Ивченко Г. И., Тимонина Е. Е. Об оценивании размера конечной совокупности. Теория вероятностей и ее применения (1982) 27, №2, 380-384.

15. Ивченко Г. И., Тимонина Е. Е. Об оптимальном оценивании для конечной совокупности, размер которой неизвестен. Матем. заметки (1982) 31, №4, 633-640.

16. Ивченко Г. И., Хонов С. А. Об асимптотическом оценивании для расслоенных конечных совокупностей. Дискретная математика (1989) 1, №3, 87-95.

17. Ивченко Г. И., Хонов С. А. Об оценивании числа классов по неполной информации. В кн.: Статистические методы оценивания и проверки гипотез Перм. ун-т, Пермь, 1993, c.52-57.

18. Козлов М. В., Прохоров А. В. Введение в математическую статистику. Изд-во МГУ, Москва, 1987.

19. Колчин В. Ф., Севастьянов Б. А., Чистяков В. П. Случайные размещения. Наука, Москва, 1976.

20. Риордан Дж. Введение в комбинаторный анализ. ИЛ, Москва, 1963. 
21. Феллер В. Введение в теорию вероятностей и ее приложения, т.1. Мир, Москва, 1964.

22. Хонов С. А. Об оценивании для расслоенной конечной совокупности в обобщенной схеме выбора // В кн.: Вероятн. прочессы и их приложения. МИЭМ, Москва, 1987, c. 41-49.

23. Хонов С. А. О несмещенном оценивании состава расслоенной конечной совокупности в обобщенной схеме выбора. В кн.: Вероятн. задачи дискретной математики. МИЭМ, Москва, 1987, с. 111-120.

24. Хонов С. А. Оценка максимального правдоподобия для размеров слоев конечной совокупности. В кн.: Вероятн. прочессы и их приложсения. МИЭМ, Москва, 1989, c. $144-147$.

25. Berg S. Factorial series distribution with applications to capture-recapture problems. Scand. J. Statist. (1974) 1, 145-152.

26. Berg S. A note on UMVU estimate in a multiple-recapture census. Scand. J. Statist. (1976) 3, 86-88.

27. Berg S. On snowball sampling, random mappings and related problems. Scand. J. Statist. (1981) 18, 283-290.

28. Driml M., Ullrich M. Maximum likelihood estimate of the number of types. Acta Techn. CSSAV. (1967) 12, №3, 300-303.

29. Good I. J. An asymptotic formula for the differences of the powers at zero. Ann. Math. Statist. (1961) 32, №1, 249-256.

30. Harris B. Statistical inference in the classical occupancy problem unbiased estimation of the number of classes. J. Amer. Statist. Assoc. (1968) 63, №323, 837-847.

31. Hodges J. L., Lehmann E. L. Some problems in minimax point estimation. Ann. Math. Statist. (1950) 21, 182-196.

32. Holst L. Some asymptotic results for incomplete multinomial or Poisson samples. Scand. J. Statist. (1981) 8, 243-246.

33. Leite J. G., Oishi J. and Pereira S. A. de B. A note on the exact maximum likelihood estimation of the size of a finite and closed population. Biometrika (1988) 75, 178-180.

34. Lewins W. A., Joanes D. N. Bayesian estimation of the number of species. Biometrics (1984) 40, №2, 323-328.

35. Murray C. M., Frees E. W. Nonparametric estimation of the probability of discovering a new species. J. Amer. Statist. Assoc. (1987) 82, №397, 305-311.

36. Seber G. A. F. A review of estimating animal abundance. Biometrics (1986) 42, 267-292.

37. Smith P. J. Bayesian methods for multiple capture-recapture surveys. Biometrics (1988) 44, 1177-1189.

38. Starr N. Linear estimation of the probability of discovering a new species. Ann. Statist. (1979) 7, №3, 644-652.

39. Trybula S. Minimax estimation of the parameters of the multivariate hypergeometric distribution. Zastos. Mat. (1985) 18, №4, 559-570.

40. Trybula S., Wilczynski M. Estimation under sampling from a finite population. Zastos. Mat. (1985) 18, №4, 571-576.

41. Wilczynski M. Minimax estimation for the multinomial and multivariate hypergeometric distribution. Sankhyã Indian J. Statist. (1985) A47, №1, 128-132.

42. Bickel P. J., Nair V. N., Wang P. C. C. Nonparametric inference under biased sampling from a finite population. Ann. Statist. (1992) 20, №2, 853-878. 